\title{
$J$-Self-Adjoint Extensions for a Class of Discrete Linear Hamiltonian Systems
}

\author{
Guojing Ren ${ }^{1}$ and Huaqing Sun ${ }^{2}$ \\ ${ }^{1}$ School of Mathematics and Quantitative Economics, Shandong University of Finance and Economics, Jinan, Shandong 250014, China \\ ${ }^{2}$ Department of Mathematics, Shandong University at Weihai, Weihai, Shandong 264209, China
}

Correspondence should be addressed to Guojing Ren; rgjmaths@gmail.com

Received 15 January 2013; Accepted 18 March 2013

Academic Editor: Michiel Bertsch

Copyright (c) 2013 G. Ren and H. Sun. This is an open access article distributed under the Creative Commons Attribution License, which permits unrestricted use, distribution, and reproduction in any medium, provided the original work is properly cited.

\begin{abstract}
This paper is concerned with formally $J$-self-adjoint discrete linear Hamiltonian systems on finite or infinite intervals. The minimal and maximal subspaces are characterized, and the defect indices of the minimal subspaces are discussed. All the $J$-self-adjoint subspace extensions of the minimal subspace are completely characterized in terms of the square summable solutions and boundary conditions. As a consequence, characterizations of all the $J$-self-adjoint subspace extensions are given in the limit point and limit circle cases.
\end{abstract}

\section{Introduction}

Consider the following discrete linear Hamiltonian system:

$$
\mathscr{J} \Delta y(t)=(P(t)+\lambda W(t)) R(y)(t), \quad t \in \mathscr{F},
$$

where $\mathscr{I}:=\{t\}_{t=a}^{b}, a$ is a finite integer or $a=-\infty, b$ is a finite integer or $b=+\infty$, and $b-a \geq 1 ; \Delta$ is the forward difference operator, that is, $\Delta y(t)=y(t+1)-y(t) ; \mathscr{J}$ is the canonical symplectic matrix, that is,

$$
\mathscr{J}=\left(\begin{array}{cc}
0 & -I_{n} \\
I_{n} & 0
\end{array}\right),
$$

where $I_{n}$ is the $n \times n$ unit matrix; the weighted function $W(t)$ is a $2 n \times 2 n$ real symmetric matrix with $W(t) \geq 0$ for $t \in \mathscr{I}$, and it is of the block diagonal form,

$$
W(t)=\operatorname{diag}\left\{W_{1}(t), W_{2}(t)\right\},
$$

where $P(t)$ is a $2 n \times 2 n$ complex symmetric matrix, that is, $P^{T}(t)=P(t)$. The partial right shift operator $R(y)(t)=\left(u^{T}(t+\right.$ $\left.1), v^{T}(t)\right)^{T}$ with $y(t)=\left(u^{T}(t), v^{T}(t)\right)^{T}$ and $u(t), v(t) \in \mathbb{C}^{n} ; \lambda$ is a complex spectral parameter.

For briefness, denote $\mathscr{I}=[a, b]$ in the case where $a$ and $b$ are finite integers; $\mathscr{I}=[a,+\infty)$ in the case where $a$ is finite and $b=+\infty ; \mathscr{I}=(-\infty, b]$ in the case where $a=-\infty$ and $b$ is finite; $\mathscr{I}=(-\infty,+\infty)$ in the case where $a=-\infty$ and $b=+\infty$.

Since $P(t)$ is symmetric, it can be blocked as

$$
P(t)=\left(\begin{array}{cc}
-C(t) & A^{T}(t) \\
A(t) & B(t)
\end{array}\right)
$$

where $A, B$, and $C$ are $n \times n$ complex-valued matrices with $C^{T}=C$ and $B^{T}=B$. Then, $\left(1_{\lambda}\right)$ can be rewritten as

$$
\begin{aligned}
&\left(I_{n}-A(t)\right) u(t+1)=u(t)+\left(B(t)+\lambda W_{2}(t)\right) v(t), \\
& v(t+1)=\left(C(t)-\lambda W_{1}(t)\right) u(t+1) \\
&+\left(I_{n}-A^{T}(t)\right) v(t), \quad t \in \mathscr{I} .
\end{aligned}
$$

To ensure the existence and uniqueness of the solution of any initial value problem for $\left(1_{\lambda}\right)$, we always assume in the present paper that

$\left(A_{1}\right) I_{n}-A(t)$ is invertible in $\mathscr{I}$. 
It can be easily verified that $\left(1_{\lambda}\right)$ contains the following complex coefficients vector difference equation of order $2 m$ :

$$
\sum_{j=0}^{m}(-1)^{j} \Delta^{j}\left[p_{j}(t) \Delta^{j} z(t-j)\right]=\lambda w(t) z(t), \quad t \in \mathscr{I},
$$

where $p_{j}(t)$ are $l \times l$ complex-valued matrices with $p_{j}^{T}(t)=$ $p_{j}(t), 0 \leq j \leq m ; p_{m}(t)$ is invertible in $\mathscr{I} ; w(t)$ is an $l \times l$ realvalued with $w(t) \geq 0$. In fact, by letting $y=\left(u^{T}, v^{T}\right)^{T}$ with $u=\left(u_{1}^{T}, u_{2}^{T}, \ldots, u_{m}^{T}\right)^{T}, v=\left(v_{1}^{T}, v_{2}^{T}, \ldots, v_{m}^{T}\right)^{T}$, and

$$
\begin{gathered}
u_{j}(t)=\Delta^{j-1} z(t-j), \\
v_{j}(t)=\sum_{k=j}^{m}(-1)^{k-j} \Delta^{k-j}\left(p_{k}(t) \Delta^{k} z(t-k)\right)
\end{gathered}
$$

for $1 \leq j \leq m,\left(3_{\lambda}\right)$ can be converted into $\left(1_{\lambda}\right)$, as well as $\left(2_{\lambda}\right)$, with

$$
\begin{gathered}
-C(t)=\operatorname{diag}\left\{p_{0}(t), p_{1}(t), \ldots, p_{m-1}(t)\right\}, \\
A(t)=\left(\begin{array}{cc}
0 & I_{l(m-1)} \\
0 & 0
\end{array}\right), \\
B(t)=\operatorname{diag}\left\{0, \ldots, 0, p_{m}^{-1}(t)\right\}, \\
W(t)=\operatorname{diag}\{w(t), 0, \ldots, 0\} .
\end{gathered}
$$

It is obvious that $\left(A_{1}\right)$ is satisfied for $\left(3_{\lambda}\right)$.

The spectral theory of self-adjoint operators and selfadjoint extensions of symmetric operators (i.e., densely defined Hermitian operators) in Hilbert spaces has been well developed (cf. [1-4]). In general, under certain definiteness conditions, a formally self-adjoint differential expression can generate a minimal operator which is symmetric, and the defect index of the minimal operator is equal to the number of linearly independent square integrable solutions. All the characterizations of self-adjoint extensions of differential equation are obtained [5-8].

However, for difference equations, it was found in [9] that the minimal operator defined in [10] may be neither densely defined nor single-valued even if the definiteness condition is satisfied. This is an important difference between the differential and difference equations. In order to study the self-adjoint extensions of nondensely defined or multivalued Hermitian operators, some scholars tried to extend the concepts and theory for densely defined Hermitian operators to Hermitian subspaces [11-15]. Recently, Shi extended the Glazman-Krein-Naimark (GKN) theory for symmetric operators to Hermitian subspaces [9]. Applying this GKN theory, the first author, with Shi and Sun, gave complete characterizations of self-adjoint extensions for second-order formally self-adjoint difference equations and general linear discrete Hamiltonian systems, separately $[16,17]$.

We note that when the coefficient $P(t)$ in $\left(1_{\lambda}\right)$ is not a Hermitian matrix, that is, $P^{*}(t) \neq P(t)$, system $\left(1_{\lambda}\right)$ is not formally self-adjoint, and the minimal subspace generated by $\left(1_{\lambda}\right)$ is not Hermitian. Hence the spectral theory of selfadjoint operators or self-adjoint subspaces is not applicable. To solve this problem, Glazman introduced a concept of $J$-symmetric operators in $[3,18]$ where $J$ is an operator. The minimal operators generated by certain differential expressions are $J$-symmetric operators in the related Hilbert spaces $[19,20]$. Monaquel and Schmidt [21] discussed the $M$ functions of the following discrete Hamiltonian system:

$$
\mathscr{J}\left(\begin{array}{l}
\Delta z_{1}(t) \\
\nabla z_{2}(t)
\end{array}\right)=(Q(t)+\lambda H(t))\left(\begin{array}{l}
z_{1}(t) \\
z_{2}(t)
\end{array}\right), \quad t \in[0,+\infty)
$$

where $\nabla$ is the backward difference operator, that is, $\nabla z(t)=$ $z(t)-z(t-1)$, and weighted function $H(t)=\operatorname{diag}\left\{H_{1}(t)\right.$, $\left.H_{2}(t)\right\}$. By letting $z_{1}(t)=v(t), z_{2}(t)=u(t+1),\left(1_{\lambda}\right)$ can be converted into $\left(4_{-\lambda}\right)$ with

$$
\begin{aligned}
& Q(t)=\left(\begin{array}{cc}
-B(t) & -A(t) \\
-A^{T}(t) & C(t)
\end{array}\right), \\
& H(t)=\left(\begin{array}{cc}
W_{2}(t) & 0 \\
0 & W_{1}(t)
\end{array}\right) .
\end{aligned}
$$

In [22], the result that every $J$-Hermitian subspace has a $J$-self-adjoint subspace extension has been given. Furthermore, a result about $J$-self-adjoint subspace extension was obtained [22], which can be regarded as a GKN theorem for $J$-Hermitian subspaces.

In the present paper, enlightened by the methods used in the study of self-adjoint subspace extensions of Hermitian subspaces, we will study the $J$-self-adjoint subspace extensions of the minimal operator corresponding to system $\left(1_{\lambda}\right)$. A complete characterization of them in terms of boundary conditions is given by employing the GKN theorem for $J$ Hermitian subspaces. The rest of this paper is organized as follows. In Section 2, some basic concepts and useful results about subspaces are briefly recalled. In Section 3, a conjugation operator $J$ is defined in the corresponding Hilbert space, and the maximal and minimal subspaces are discussed. In Section 4, the description of the minimal subspaces is given by the properties of their elements at the endpoints of the discussed intervals, the defect indices of minimal subspaces are discussed, and characterizations of the maximal subspaces are established. Section 5 pays attention to two characterizations of all the self-adjoint subspace extensions of the minimal subspace in terms of boundary conditions via linearly independent square summable solutions of $\left(1_{\lambda}\right)$. As a consequence, characterizations of all the self-adjoint subspace extensions are given in two special cases: the limit point and limit circle cases.

\section{Fundamental Results on Subspaces}

In this section, we recall some basic concepts and useful results about subspaces. For more results about nondensely defined $J$-Hermitian operators or $J$-Hermitian subspaces, we refer to $[17-19,22]$ and some references cited therein. In addition, some properties of solutions of $\left(1_{\lambda}\right)$ and a result about matrices are given at the end of this section. 
By $\mathbb{R}$ and $\mathbb{C}$ we denote the sets of the real and the complex numbers, respectively. Let $X$ be a complex Hilbert space equipped with inner product $\langle\cdot, \cdot\rangle, T$ and $S$ two linear subspaces (briefly, subspace) in $X^{2}:=X \times X$, and $\lambda \in \mathbb{C}$. Denote

Dom $T:=\{x \in X:(x, f) \in T$ for some $f \in X\}$,

Ran $T:=\{f \in X:(x, f) \in T$ for some $x \in X\}$,

$\operatorname{Ker} T:=\{x \in X:(x, 0) \in T\}$,

$$
\begin{aligned}
T^{*}:= & \left\{(x, f) \in X^{2}:\langle x, g\rangle=\langle f, y\rangle \forall(y, g) \in T\right\}, \\
& T-\lambda I:=\{(x, f-\lambda x):(x, f) \in T\} .
\end{aligned}
$$

If $T \cap S=\{0\}$, we write

$$
T+S:=\{(x+y, f+g):(x, f) \in T,(y, g) \in S\},
$$

which is denoted by $T \oplus S$ in the case that $T$ and $S$ are orthogonal.

Denote

$$
T(x):=\{f \in X:(x, f) \in T\} .
$$

It can be easily verified that $T(0)=\{0\}$ if and only if $T$ can determine a unique linear operator from Dom $T$ into $X$ whose graph is just $T$. For convenience, we will identify a linear operator in $X$ with a subspace in $X^{2}$ via its graph.

Definition 1 (see [11]). Let $T$ be a subspace in $X^{2}$.

(1) $T$ is said to be a Hermitian subspace if $T \subset T^{*}$. Furthermore, $T$ is said to be a Hermitian operator if it is an operator, that is, $T(0)=\{0\}$.

(2) $T$ is said to be a self-adjoint subspace if $T=T^{*}$. Furthermore, $T$ is said to be a self-adjoint operator if it is an operator, that is, $T(0)=\{0\}$.

(3) Let $T$ be a Hermitian subspace. $T_{1}$ is said to be a selfadjoint subspace extension (briefly, SSE) of $T$ if $T \subset T_{1}$ and $T_{1}$ is a self-adjoint subspace.

(4) Let $T$ be a Hermitian operator. $T_{1}$ is said to be a selfadjoint operator extension (briefly, SOE) of $T$ if $T \subset$ $T_{1}$ and $T_{1}$ is a self-adjoint operator.

Lemma 2 (see [11]). Let $T$ be a subspace in $X^{2}$. Then

(1) $T^{*}$ is a closed subspace in $X^{2}$;

(2) $T^{*}=(\bar{T})^{*}$ and $T^{* *}=\bar{T}$, where $\bar{T}$ is the closure of $T$;

(3) $\operatorname{Ker} T^{*}=(\operatorname{Ran} T)^{\perp}=(\operatorname{Ran} \bar{T})^{\perp}$.

In [19], an operator $J$ defined in $X$ is said to be a conjugation operator if for all $x, y \in X$,

$$
\langle J x, J y\rangle=\langle y, x\rangle, \quad J^{2} x=x .
$$

Definition 3. Let $T$ be a subspace in $X^{2}$ and $J$ be a conjugation operator.
(1) The $J$-adjoint of $T$ is defined by

$T_{J}^{*}:=\left\{(y, g) \in X^{2}:\langle f, J y\rangle=\langle x, J g\rangle \forall(x, f) \in T\right\}$.

(2) $T$ is said to be a $J$-Hermitian subspace if $T \subset T_{J}^{*}$. Furthermore, $T$ is said to be a $J$-Hermitian operator if it is an operator, that is, $T(0)=\{0\}$.

(3) $T$ is said to be a $J$-self-adjoint subspace if $T=T_{J}^{*}$. Furthermore, $T$ is said to be a $J$-self-adjoint operator if it is an operator, that is, $T(0)=\{0\}$.

(4) Let $T$ be a $J$-Hermitian subspace. $T_{1}$ is said to be a $J$ self-adjoint subspace extension (briefly, $J$-SSE) of $T$ if $T \subset T_{1}$ and $T_{1}$ is a $J$-self-adjoint subspace.

(5) Let $T$ be a $J$-Hermitian operator. $T_{1}$ is said to be a $J$ self-adjoint operator extension (briefly, $J$-SOE) of $T$ if $T \subset T_{1}$ and $T_{1}$ is a $J$-self-adjoint operator.

Remark 4. (1) It can be easily verified that $T_{J}^{*}$ is a closed subspace. Consequently, a $J$-self-adjoint subspace $T$ is a closed subspace since $T=T_{J}^{*}$. In addition, $S_{J}^{*} \subset T_{J}^{*}$ if $T \subset S$.

(2) From the definition, we have that $\langle f, J y\rangle=\langle x, J g\rangle$ holds for all $(x, f) \in T$ and $(y, g) \in T_{J}^{*}$, and that $T$ is a $J$ Hermitian subspace if and only if $\langle f, J y\rangle=\langle x, J g\rangle$ for all $(x, f),(y, g) \in T$.

Lemma 5 (see [22]). Let $T$ be a subspace in $X^{2}$. Then

(1) $T^{*}:=\left\{(J y, J g):(y, g) \in T_{J}^{*}\right\}$;

(2) $T_{J}^{*}:=\left\{(J y, J g):(y, g) \in T^{*}\right\}$.

It follows from Lemmas 2 and 5 that $T_{J}^{*}=(\bar{T})_{J}^{*}$, and $\bar{T}$ is $J$-Hermitian if $T$ is $J$-Hermitian.

Lemma 6 (see [22]). Every J-Hermitian subspace has a J-SSE.

Definition 7. Let $\mathrm{T}$ be a $J$-Hermitian subspace. Then $d(T)=$ $(1 / 2) \operatorname{dim} T_{J}^{*} / \bar{T}$ is called to be the defect index of $T$.

Next, we introduce a form on $X^{2} \times X^{2}$ by

$$
\begin{aligned}
{[(x, f):(y, g)]:=} & \langle f, J y\rangle-\langle x, J g\rangle \\
& (x, f),(y, g) \in X^{2} .
\end{aligned}
$$

Lemma 8 (see [22]). Let $T$ be a J-Hermitian subspace. Then

$$
\bar{T}=\left\{(x, f) \in T_{J}^{*}:[(x, f):(y, g)]=0, \forall(y, g) \in T_{J}^{*}\right\} .
$$

Lemma 9 (see [22]). Let $T$ be a closed J-Hermitian subspace in $X^{2}$ and satisfy $d=d(T)<+\infty$. Then a subspace $T_{1}$ is a J-SSE of $T$ if and only if $T \subset T_{1} \subset T_{J}^{*}$ and there exists $\left\{\left(x_{j}, f_{j}\right)\right\}_{j=1}^{d} \subset$ $T_{J}^{*}$ such that

(1) $\left(x_{1}, f_{1}\right),\left(x_{2}, f_{2}\right), \ldots,\left(x_{d}, f_{d}\right)$ are linearly independent in $T_{J}^{*}$ (modulo $\left.T\right)$;

(2) $\left[\left(x_{j}, f_{j}\right):\left(x_{k}, f_{k}\right)\right]=0,1 \leq j, k \leq d$; 
(3) $T_{1}=\left\{(y, g) \in T_{J}^{*}:\left[(y, g):\left(x_{j}, f_{j}\right)\right]=0,1 \leq j \leq d\right\}$.

Lemma 9 can be regarded as a GKN theorem for $J$ Hermitian subspaces. A set of $\left\{\left(x_{j}, f_{j}\right)\right\}_{j=1}^{d} \subset T_{J}^{*}$ which is satisfying (1) and (2) in Lemma 9 is called a GKN set of $T$.

Definition 10. Let $T$ be a subspace in $X^{2}$.

(1) The set

$$
\rho(T):=\left\{\lambda \in \mathbb{C}:(T-\lambda)^{-1}\right.
$$

is a bounded linear operator defined in $X\}$

is called the resolvent set of $T$.

(2) The set $\sigma(T):=\mathbb{C} \backslash \rho(T)$ is called the spectrum of $T$.

(3) The set

$$
\begin{aligned}
\Gamma(T):=\{\lambda \in \mathbb{C}: \exists c(\lambda)>0 \text { s.t. } \\
\\
\|f-\lambda x\| \geq c(\lambda)\|x\|, \quad \forall(x, f) \in T\}
\end{aligned}
$$

is called to be the regularity field of $T$.

It is evident that $\rho(T) \subset \Gamma(T)$ for any subspace $T$ in $X^{2}$.

Lemma 11 (see [22]). Let $T$ be a J-Hermitian subspace in $X^{2}$ with $\Gamma(T) \neq \emptyset$, and $\lambda \in \Gamma(T)$. Then

$$
\begin{gathered}
T_{J}^{*}=\bar{T}+\left\{(y, g) \in T_{J}^{*}: g-\lambda y \in \operatorname{Ker}\left(T^{*}-\bar{\lambda}\right)\right\}, \\
d(T)=\operatorname{dim} \operatorname{Ran}(T-\lambda)^{\perp}=\operatorname{dim} \operatorname{Ker}\left(T^{*}-\bar{\lambda}\right) .
\end{gathered}
$$

The following is a well-known result on the rank of matrices.

Lemma 12. Let $A$ be an $m \times l$ matrix and $B$ an $l \times n$ matrix. Then

$$
\begin{aligned}
\operatorname{rank} A+\operatorname{rank} B-l & \leq \operatorname{rank}(A B) \\
& \leq \min \{\operatorname{rank} A, \operatorname{rank} B\} .
\end{aligned}
$$

In particular, if $A B=0$, then

$$
\text { rank } A+\operatorname{rank} B \leq l .
$$

\section{Relationship between the Maximal and Minimal Subspaces}

This section is divided into three subsections. In the first subsection, we define a conjugation operator in a Hilbert space. In the second subsection, we define maximal and minimal subspaces generated by $\left(1_{\lambda}\right)$ and discuss relationship between them. In the last subsection, we discuss the definiteness condition corresponding to $\left(1_{\lambda}\right)$.
3.1. Conjugation Operator. In this subsection, we define a conjugation operator in a Hilbert space and then discuss its properties.

Since $b$ and $a$ may be finite or infinite, we introduce the following conventions for briefness: $b+1$ means $+\infty$ in the case of $b=+\infty$ and $a-1$ means $-\infty$ in the case of $a=-\infty$. Denote

$$
\begin{gathered}
\mathscr{I}^{*}:=\{t\}_{t=a}^{b+1}, \\
l(\mathscr{I}):=\left\{y: y=\{y(t)\}_{t \in \mathcal{I}^{*}} \subset \mathbb{C}^{2 n}\right\} .
\end{gathered}
$$

For any $2 n \times 2 n$ Hermitian matrix $W(t) \geq 0$ defined in $\mathscr{I}$, we define

$$
\begin{aligned}
& \mathscr{L}_{W}^{2}(\mathscr{I}) \\
& \quad:=\left\{y \in l(\mathscr{I}): \sum_{t \in \mathscr{I}} R(y)^{*}(t) W(t) R(y)(t)<+\infty\right\}
\end{aligned}
$$

with the semiscalar product

$$
\langle y, z\rangle:=\sum_{t \in \mathscr{I}} R^{*}(z)(t) W(t) R(y)(t) .
$$

Furthermore, denote $\|y\|:=(\langle y, y\rangle)^{1 / 2}$ for $y \in \mathscr{L}_{W}^{2}(\mathscr{I})$. Since the weighted function $W(t)$ may be singular in $\mathscr{F},\|\cdot\|$ is a seminorm. Introduce the quotient space

$$
L_{W}^{2}(\mathscr{I}):=\frac{\mathscr{L}_{W}^{2}(\mathscr{I})}{\left\{y \in \mathscr{L}_{W}^{2}(\mathscr{I}):\|y\|=0\right\}}
$$

Then $L_{W}^{2}(\mathscr{I})$ is a Hilbert space with the inner product $\langle\cdot, \cdot\rangle$.

For a function $y \in \mathscr{L}_{W}^{2}(\mathscr{I})$, denote by $y^{\pi}$ the corresponding class in $L_{W}^{2}(\mathscr{I})$. And for any $y^{\pi} \in L_{W}^{2}(\mathscr{I})$, denote by $y \in$ $\mathscr{L}_{W}^{2}(\mathscr{I})$ a representative of $y^{\pi}$. It is evident that $\left\langle y^{\pi}, z^{\pi}\right\rangle=$ $\langle y, z\rangle$ for any $y^{\pi}, z^{\pi} \in L_{W}^{2}(\mathscr{I})$.

For any $y \in \mathscr{L}_{W}^{2}(\mathscr{F})$, denote by $J_{0} y$ the conjugation of $y$; that is,

$$
J_{0} y:=\{\bar{y}(t)\}_{t \in \mathscr{I}^{*}}, \quad y \in \mathscr{L}_{W}^{2}(\mathscr{T}) .
$$

It can be easily verified that $y \in \mathscr{L}_{W}^{2}(\mathscr{I})$ if and only if $J_{0} y \epsilon$ $\mathscr{L}_{\bar{W}}^{2}(\mathscr{I})$. Here $\bar{W}$ is the conjugation of matrix $W$. Since each $y^{\pi} \in L_{W}^{2}(\mathscr{I})$ is an equivalent class, we define a operator $J$ defined on $L_{W}^{2}(\mathscr{I})$ by

$$
J\left(y^{\pi}\right):=\left(J_{0} y\right)^{\pi}, \quad y^{\pi} \in L_{W}^{2}(\mathscr{I}) .
$$

The following result is obtained.

Lemma 13. J defined by (24) is a conjugation operator defined on $L_{W}^{2}(\mathscr{F})$ if and only if $W(t)$ is real and symmetric in $\mathscr{I}$.

Proof. The sufficiency is evident. Next, we consider the necessity. Assume that $J$ defined by (24) is a conjugation operator in 
$L_{W}^{2}(\mathscr{I})$. Then for any $x^{\pi}, y^{\pi} \in L_{W}^{2}(\mathscr{I})$, it follows from $\left\langle J x^{\pi}\right.$, $\left.J y^{\pi}\right\rangle=\left\langle y^{\pi}, x^{\pi}\right\rangle$ that

$$
\sum_{t \in \mathscr{I}} R(x)^{*}(t) W^{T}(t) R(y)(t)=\sum_{t \in \mathscr{I}} R(x)^{*}(t) W(t) R(y)(t)
$$

By the arbitrariness of $x^{\pi}, y^{\pi}$, one has that $W(t)=W^{T}(t)$. This, together with $W(t)=W^{*}(t)$, yields that $W(t)$ is real. The proof is complete.

For any $x, y \in l(\mathscr{I})$, we denote

$$
(x, y)(t)=y^{T}(t) \mathscr{J} x(t), \quad t \in \mathscr{I}^{*},
$$

where $\mathscr{J}$ is the canonical symplectic matrix given in Section 1 . In the case of $b=+\infty$, if $\lim _{t \rightarrow b}(x, y)(t)$ exists and is finite, then its limit is denoted by $(x, y)(+\infty)$. In the case of $a=-\infty$, if $\lim _{t \rightarrow a}(x, y)(t)$ exists and is finite, then its limit is denoted by $(x, y)(-\infty)$.

Denote

$$
\begin{aligned}
& \tau(y)(t):=\mathscr{J} \Delta y(t)-P(t) R(y)(t), \\
& \delta(y)(t):=\mathscr{J} \Delta y(t)-\bar{P}(t) R(y)(t),
\end{aligned}
$$

where $\tau$ and $\delta$ are called the natural difference operators corresponding to system $\left(1_{\lambda}\right)$. The following result can be easily verified, and so we omit the proof.

Lemma 14. Assume that $\left(A_{1}\right)$ holds. Let $x, y \in l(\mathscr{I})$.

(1) $\delta\left(J_{0} y\right)=J_{0} \tau(y), \tau\left(J_{0} y\right)=J_{0} \delta(y)$.

(2) For any $s, k \in \mathscr{I}$,

$$
\begin{aligned}
\sum_{t=s}^{k} & {\left[R\left(J_{0} y\right)^{*}(t) \tau(x)(t)\right.} \\
& \left.-\left(J_{0} \tau(y)\right)^{*}(t) R(x)(t)\right] \\
= & \left.(x, y)(t)\right|_{s} ^{k+1} .
\end{aligned}
$$

(3) For any $\lambda \in \mathbb{C}, c_{0} \in \mathscr{I}$, and any two solutions $x(t)$ and $y(t)$ of $\left(1_{\lambda}\right)$, it follows that

$$
(x, y)(t)=(x, y)\left(c_{0}\right), \quad t \in \mathscr{I}^{*} .
$$
then

Moreover, let $Y(t, \lambda)$ be a fundamental solution of $\left(1_{\lambda}\right)$,

$$
Y^{T}(t, \lambda) \mathscr{J} Y(t, \lambda)=Y^{T}\left(c_{0}, \lambda\right) \mathscr{J} Y\left(c_{0}, \lambda\right), \quad t \in \mathscr{I}^{*}
$$

3.2. Relationship between the Maximal and Minimal Subspaces. In this subsection, we first introduce the maximal and minimal subspaces corresponding to $\left(1_{\lambda}\right)$ and then show that the minimal subspace is $J$-Hermitian, and its $J$-adjoint subspace is just the maximal subspace.
Denote

$\mathscr{L}_{W, 0}^{2}(\mathscr{I})$

$:=\left\{y \in \mathscr{L}_{W}^{2}(\mathscr{I}):\right.$ there exist two integers $s, k \in \mathscr{I}$

with $s \leq k$ such that $y(t)=0$ for $t \leq s$ and $t \geq k+1\}$,

and define

$$
\begin{gathered}
H(\tau):=\left\{\left(y^{\pi}, g^{\pi}\right) \in\left(L_{W}^{2}(\mathscr{I})\right)^{2}: \exists y \in y^{\pi}\right. \\
\text { s.t. }(y)(t)=W(t) R(g)(t) \text { in } \mathscr{I}\}, \\
H_{00}(\tau):=\left\{\left(y^{\pi}, g^{\pi}\right) \in H(\tau): \exists y \in y^{\pi} \text { s.t. } y \in \mathscr{L}_{W, 0}^{2}(\mathscr{I})\right. \text { and } \\
\\
\tau(y)(t)=W(t) R(g)(t) \text { in } \mathscr{I}\} .
\end{gathered}
$$

It can be easily verified that $H(\tau)$ and $H_{00}(\tau)$ are both linear subspaces in $\left(L_{W}^{2}(\mathscr{I})\right)^{2}$. Here, $H(\tau)$ and $H_{00}(\tau)$ are called the maximal and preminimal subspaces corresponding to $\tau$ or $\left(1_{\lambda}\right)$ in $\left(L_{W}^{2}(\mathscr{I})\right)^{2}$, and $H_{0}(\tau):=\bar{H}_{00}(\tau)$ is called the minimal subspace corresponding to $\tau$ or $\left(1_{\lambda}\right)$ in $\left(L_{W}^{2}(\mathscr{I})\right)^{2}$.

Since the end points $a$ and $b$ may be finite or infinite, we need to divide $\mathscr{I}$ into two subintervals in order to characterize the maximal and minimal subspaces in a unified form. Choose $a<c_{0}<b$ and fix it. Denote

$$
\mathscr{I}_{a}:=\{t\}_{t=a}^{c_{0}-1}, \quad \mathscr{I}_{b}:=\{t\}_{t=c_{0}}^{b},
$$

and denote by $\langle\cdot, \cdot\rangle_{a},\langle\cdot, \cdot\rangle_{b}$ and $\|\cdot\|_{a},\|\cdot\|_{b}$ the inner products and norms of $\mathscr{L}_{W}^{2}\left(\mathscr{I}_{a}\right), \mathscr{L}_{W}^{2}\left(\mathscr{I}_{b}\right)$, respectively. Let $\mathscr{L}_{W, 0}^{2}\left(\mathscr{I}_{a}\right)$ and $\mathscr{L}_{W, 0}^{2}\left(\mathscr{I}_{b}\right)$ be defined by (31) with $\mathscr{I}$ replaced by $\mathscr{I}_{a}$ and $\mathscr{I}_{b}$, respectively. Furthermore, let $H_{a}(\tau)$ and $H_{a, 00}(\tau)$ be the left maximal and preminimal subspaces defined by (32) with $\mathscr{I}$ replaced by $\mathscr{I}_{a}$, respectively, and $H_{b}(\tau)$ and $H_{b, 00}(\tau)$ the right maximal and preminimal subspaces defined by (32) with $\mathscr{I}$ replaced by $\mathscr{I}_{b}$, respectively. The subspaces $H_{a, 0}(\tau):=\bar{H}_{a, 00}(\tau)$ and $H_{b, 0}(\tau):=\bar{H}_{b, 00}(\tau)$ are called the left and right minimal subspaces corresponding to system $\left(1_{\lambda}\right)$ in $\mathscr{I}_{a}$ and $\mathscr{I}_{b}$, respectively. Similarly, we can define $H(\delta), H_{00}(\delta)$, and $H_{0}(\delta) ; H_{b}(\delta), H_{b, 00}(\delta)$, and $H_{b, 0}(\delta) ; H_{a}(\delta)$, $H_{a, 00}(\delta)$ and $H_{a, 0}(\delta)$.

The following result is directly derived from (1) of Lemma 14

Lemma 15. Assume that $\left(A_{1}\right)$ holds. Then $\left(y^{\pi}, g^{\pi}\right) \in H(\tau)$ if and only if $\left(J y^{\pi}, J g^{\pi}\right) \in H(\delta)$.

In order to study properties of the above subspaces, we first make some preparation.

Let $Y(t)$ be the fundamental solution matrix of $\left(1_{0}\right)$ with $Y\left(c_{0}\right)=I_{2 n}$. For any finite subinterval $\mathscr{I}^{\prime}$ with $c_{0} \in \mathscr{I}^{\prime} \subset \mathscr{I}$, denote

$$
\Phi_{\mathscr{I}^{\prime}}:=\sum_{t \in \mathscr{I}^{\prime}} R(Y)^{*}(t) W(t) R(Y)(t)
$$


It is evident that $\Phi_{\mathscr{J}^{\prime}}$ is a $2 n \times 2 n$ positive semidefinite matrix and dependent on $\mathscr{I}^{\prime}$. By the same method used in [23, Lemma 3.2], it follows that there exists a finite subinterval $\mathscr{I}_{0}$ with $c_{0} \in \mathscr{I}_{0} \subset \mathscr{I}$ such that

$$
\operatorname{rank} \Phi_{\mathscr{I}_{0}}=\operatorname{rank} \Phi_{\mathscr{I}^{\prime}}, \quad \operatorname{Ran} \Phi_{\mathscr{J}_{0}}=\operatorname{Ran} \Phi_{\mathscr{I}^{\prime}},
$$

for any finite subinterval $\mathscr{I}^{\prime}$ with $\mathscr{I}_{0} \subset \mathscr{I}^{\prime} \subset \mathscr{I}$. In the present paper, we will always denote $\mathscr{I}_{0}:=\left[s_{0}, t_{0}\right]$ and define

$$
\operatorname{rank} \Phi_{\mathscr{I}}:=\operatorname{rank} \Phi_{\mathscr{I}_{0}}, \quad \operatorname{Ran} \Phi_{\mathscr{I}}:=\operatorname{Ran} \Phi_{\mathscr{I}_{0}},
$$

whenever $\mathscr{I}$ is finite or infinite. In the case that $\mathscr{I}$ is finite, $\mathscr{I}_{0}$ can be taken as $\mathscr{I}$.

In the case that $\mathscr{I}$ is finite, we define

$$
\begin{aligned}
& \phi_{\mathscr{I}}: L_{W}^{2}(\mathscr{I}) \longrightarrow \mathbb{C}^{2 n}, \\
& g^{\pi} \longmapsto \sum_{s \in \mathscr{I}} R\left(J_{0} Y\right)^{*}(s) W(s) R(g)(s) .
\end{aligned}
$$

It is evident that $\phi_{\mathscr{I}}$ is a bounded linear map and its range is a closed subset in $\mathbb{C}^{2 n}$.

In the case that $\mathscr{I}$ is infinite, that is, $\mathscr{I}=[a,+\infty)$ or $\mathscr{I}=$ $(-\infty, b]$ or $\mathscr{I}=(-\infty,+\infty)$, where $a, b$ are finite integers, we introduce the following subspaces of $L_{W}^{2}(\mathscr{I})$, respectively:

$$
\begin{aligned}
& L_{W, 1}^{2}(\mathscr{I}) \\
& :=\left\{y^{\pi} \in L_{W}^{2}(\mathscr{I}): \exists k \in \mathscr{I}\right. \\
& \text { s.t. } W(t) R(y)(t)=0 \text { for } t \geq k\} \text {, } \\
& L_{W, 1}^{2}(\mathscr{I}) \\
& :=\left\{y^{\pi} \in L_{W}^{2}(\mathscr{I}): \exists s \in \mathscr{I}\right. \\
& \text { s.t. } W(t) R(y)(t)=0 \text { for } t \leq s\}, \\
& L_{W, 1}^{2}(\mathscr{I}) \\
& :=\left\{y^{\pi} \in L_{W}^{2}(\mathscr{I}): \exists s \leq k \in \mathscr{I}\right. \\
& \text { s.t. } W(t) R(y)(t)=0 \text { for } t \leq s \text { and } t \geq k\} \text {. }
\end{aligned}
$$

It can be easily shown that $L_{W, 1}^{2}(\mathscr{I})$ is dense in $L_{W}^{2}(\mathscr{I})$. In this case, we define

$$
\begin{aligned}
& \phi_{\mathscr{I}}: L_{W, 1}^{2}(\mathscr{I}) \longrightarrow \mathbb{C}^{2 n}, \\
& g^{\pi} \longmapsto \sum_{s \in \mathscr{I}} R\left(J_{0} Y\right)^{*}(s) W(s) R(g)(s) .
\end{aligned}
$$

By the method used in [23, Lemma 3.3], one has the following properties of $\phi_{\mathscr{I}}$.

Lemma 16. Assume that $\left(A_{1}\right)$ holds.

(1) $\operatorname{Ran} \phi_{\mathscr{I}}=\operatorname{Ran} \Phi_{\mathscr{I}}$.
(2) In the case that $\mathscr{I}$ is finite,

$L_{W}^{2}(\mathscr{I})=\operatorname{Ker} \phi_{\mathscr{I}} \oplus\left\{\left(J_{0}(Y \xi)\right)^{\pi}: \xi \in \operatorname{Ran} \Phi_{\mathscr{I}}\right\} ;$

in the case that $\mathscr{I}$ is infinite, let $l=\operatorname{rank} \Phi_{\mathscr{I}}$. Then there exist linearly independent elements $h_{j}^{\pi} \in$ $L_{W, 1}^{2}(\mathscr{I}), 1 \leq j \leq l$, such that

$$
L_{W, 1}^{2}(\mathscr{I})=\operatorname{Ker} \phi_{\mathscr{I}} \dot{+} \operatorname{span}\left\{h_{1}^{\pi}, h_{2}^{\pi}, \ldots, h_{l}^{\pi}\right\} .
$$

(3) $\operatorname{Ker} \phi_{\mathscr{J}} \subset \operatorname{Ran}\left(H_{00}(\tau)\right)$.

The following is the main result of this section.

Theorem 17. Assume that $\left(A_{1}\right)$ holds. Then $H(\tau)=$ $\left(H_{00}(\tau)\right)_{J}^{*}=\left(H_{0}(\tau)\right)_{J}^{*}, H_{b}(\tau)=\left(H_{b, 00}(\tau)\right)_{J}^{*}=\left(H_{b, 0}(\tau)\right)_{J}^{*}$, and $H_{a}(\tau)=\left(H_{a, 00}(\tau)\right)_{J}^{*}=\left(H_{a, 0}(\tau)\right)_{J}^{*}$.

Proof. Since the method of the proofs is similar, we only show the first assertion. By $\left(H_{0}(\tau)\right)_{J}^{*}=\left(H_{00}(\tau)\right)_{J}^{*}$, it suffices to show $H(\tau)=\left(H_{00}(\tau)\right)_{J}^{*}$.

We first show that $H(\tau) \subset\left(H_{00}(\tau)\right)_{J}^{*}$. Let $\left(y^{\pi}, g^{\pi}\right) \in H(\tau)$. Then for any $\left(x^{\pi}, f^{\pi}\right) \in H_{00}(\tau)$, there exists $x \in x^{\pi}$ with $x \in$ $\mathscr{L}_{W}^{2}(\mathscr{I})$ such that $\tau(x)(t)=W(t) R(f)(t)$ in $\mathscr{I}$. So, it follows from (2) of Lemma 14 that

$$
\begin{gathered}
\left\langle f^{\pi}, J y^{\pi}\right\rangle-\left\langle x^{\pi}, J g^{\pi}\right\rangle \\
=\sum_{t \in \mathscr{I}}\left[R\left(J_{0} y\right)^{*}(t) W(t) R(f)(t)\right. \\
\left.-R\left(J_{0} g\right)^{*}(t) W(t) R(x)(t)\right] \\
=\sum_{t \in \mathscr{I}}\left[R\left(J_{0} y\right)^{*}(t) \tau(x)(t)\right. \\
\left.\quad-\left(J_{0} \tau(y)\right)^{*}(t) R(x)(t)\right] \\
=\left.(x, y)(t)\right|_{a} ^{b+1}=0 .
\end{gathered}
$$

This implies that $H(\tau) \subset\left(H_{00}(\tau)\right)_{J}^{*}$.

Next, we show $\left(H_{00}(\tau)\right)_{J}^{*} \subset H(\tau)$. Fix any $\left(y^{\pi}, g^{\pi}\right) \in$ $\left(H_{00}(\tau)\right)_{J}^{*}$. It suffices to show that there exists $y_{0} \in y^{\pi}$ such that $\tau\left(y_{0}\right)(t)=W(t) R(g)(t)$ in $\mathscr{I}$. Let $z$ be a solution of $\tau(z)(t)=W(t) R(g)(t)$ on $\mathscr{I}$. For any $\left(x^{\pi}, f^{\pi}\right) \in H_{00}(\tau)$, there exits $x \in x^{\pi}$ with $x \in \mathscr{L}_{W, 0}^{2}(\mathscr{I})$ such that $\tau(x)(t)=$ $W(t) R(f)(t)$ in $\mathscr{I}$. Thus, it follows from (2) of Lemma 14 that

$$
\begin{aligned}
\sum_{t \in \mathscr{I}}\left[R\left(J_{0} z\right)^{*}(t) W(t) R(f)(t)\right. \\
\left.\quad-R\left(J_{0} g\right)^{*}(t) W(t) R(x)(t)\right] \\
\left.=\sum_{t \in \mathscr{I}}\left[R\left(J_{0} z\right)^{*}(t) \tau(x)(t)-\left(J_{0} \tau(z)\right)^{*}(t)\right) R(x)(t)\right] \\
=\left.(x, z)(t)\right|_{a} ^{b+1}=0 .
\end{aligned}
$$


In addition, it is clear that

$$
\begin{aligned}
\left\langle f^{\pi}, J y^{\pi}\right\rangle-\left\langle x^{\pi}, J g^{\pi}\right\rangle=\sum_{t \in \mathscr{F}}[ & R\left(J_{0} y\right)^{*}(t) W(t) R(f)(t) \\
& \left.-R\left(J_{0} g\right)^{*}(t) W(t) R(x)(t)\right]
\end{aligned}
$$

$=0$.

Combining (45) and (46), one has that for all $\left(x^{\pi}, f^{\pi}\right) \in$ $H_{00}(\tau)$,

$$
\sum_{t \in \mathscr{F}} R\left(J_{0}(y-z)\right)^{*}(t) W(t) R(f)(t)=0
$$

By (2) and (3) of Lemma 16, we get that for any $h^{\pi} \in \operatorname{Ker} \phi_{\mathscr{I}}$ and any $\xi \in \mathbb{C}^{2 n}$

$$
\sum_{t \in \mathscr{I}} R\left(J_{0}(y-z-Y \xi)\right)^{*}(t) W(t) R(h)(t)=0 .
$$

The following discussion is divided into two parts.

Case 1. $\mathscr{I}$ is finite. It is evident that $y^{\pi}, z^{\pi} \in L_{W}^{2}(\mathscr{I})$. Then, from (2) of Lemma 16, there exists $\xi_{0} \in \operatorname{Ran} \Phi_{\mathscr{I}}$ such that $\left(J_{0}\left(y-z-Y \xi_{0}\right)\right)^{\pi} \in \operatorname{Ker} \phi_{g}$. This, together with (48), implies that $\left(J_{0}\left(y-z-Y \xi_{0}\right)\right)^{\pi}=0$. This is equivalent to $\left(y-z-Y \xi_{0}\right)^{\pi}=$ 0 . Let $y_{0}(t):=z(t)+Y(t) \xi_{0}$. Then $y_{0} \in y^{\pi}$ and satisfies

$$
\tau\left(y_{0}\right)(t)=\tau(z)(t)+\tau(Y \xi)(t)=W(t) R(g)(t), \quad t \in \mathscr{I} .
$$

Hence, $\left(y^{\pi}, g^{\pi}\right) \in H(\tau)$. Since $\left(y^{\pi}, g^{\pi}\right) \in H_{00}^{*}(\tau)$ is arbitrary, we have $\left(H_{00}(\tau)\right)_{J}^{*} \subset H(\tau)$.

Case 2. $\mathscr{I}$ is infinite. We only consider the case that $\mathscr{I}=$ $[a,+\infty)$. For the other two cases, it can be proved with similar arguments.

Let rank $\Phi_{\mathscr{I}}=l$. With a similar argument as Case 2 of the proof of [23, Theorem 3.1], it can be shown that there exist linearly independent elements $h_{j}^{\pi} \in L_{W, 1}^{2}, 1 \leq j \leq l$, and $\xi_{0} \in \operatorname{Ran} \Phi_{\mathscr{I}}$ such that $\left(y-z-Y \xi_{0}\right)^{\pi} \in \operatorname{Ker} \phi_{\mathscr{F}}$,

$$
\begin{gathered}
L_{W, 1}^{2}(\mathscr{I})=\operatorname{Ker} \phi_{\mathscr{I}} \dot{+} \operatorname{span}\left\{h_{1}^{\pi}, h_{2}^{\pi}, \ldots, h_{l}^{\pi}\right\}, \\
\sum_{t \in \mathscr{I}} R\left(J_{0}\left(y-z-Y \xi_{0}\right)\right)^{*}(t) W(t) R\left(h_{j}\right)(t)=0, \quad 1 \leq j \leq l .
\end{gathered}
$$

Combining (48)-(50), one has that for any $h^{\pi} \in L_{W, 1}^{2}(\mathscr{I})$

$$
\sum_{t \in \mathscr{I}} R\left(J_{0}\left(y-z-Y \xi_{0}\right)\right)^{*}(t) W(t) R(h)(t)=0 .
$$

This implies that $\left(y-z-Y \xi_{0}\right)^{\pi}=0$ and consequently $y_{0}:=$ $z+Y \xi_{0}$ is a representative of $y^{\pi}$ such that $\tau\left(y_{0}\right)(t)=$ $W(t) R(g)(t)$. So $\left(y^{\pi}, g^{\pi}\right) \in H(\tau)$. By the arbitrariness of $\left(y^{\pi}\right.$, $\left.g^{\pi}\right)$ one has $\left(H_{00}(\tau)\right)_{J}^{*} \subset H(\tau)$.

The entire proof is complete.
The following result is directly derived from Lemmas 5 and 15 , and Theorem 17.

Theorem 18. Assume that $\left(A_{1}\right)$ holds. Then $H(\delta)=$ $\left(H_{00}(\tau)\right)^{*}, H_{b}(\delta)=\left(H_{b, 00}(\tau)\right)^{*}$, and $H_{a}(\delta)=\left(H_{a, 00}(\tau)\right)^{*}$.

3.3. Definiteness Condition. In this subsection, we introduce the definiteness condition for $\left(1_{\lambda}\right)$, and give some important results on it. Since the proofs are similar to those given in [23], we omit the proofs.

The definiteness condition for $\left(1_{\lambda}\right)$ or $H(\tau)$ is given by the following.

$\left(A_{2}\right)$ There exists a finite subinterval $\mathscr{I}_{1} \subset \mathscr{I}$ such that for any $\lambda \in \mathbb{C}$ and for any nontrivial solution $y(t)$ of $\left(1_{\lambda}\right)$, the following always holds:

$$
\sum_{t \in \mathscr{F}_{1}} R(y)^{*}(t) W(t) R(y)(t)>0 .
$$

In particular, the definiteness condition for $\left(3_{\lambda}\right)$ can be described as there exists a finite subinterval $\mathscr{I}_{1} \subset \mathscr{I}$ such that for any $\lambda \in \mathbb{C}$ and for any nontrivial solution $z(t)$ of $\left(3_{\lambda}\right)$, the following always holds:

$$
\sum_{t \in \mathscr{I}_{1}} z^{*}(t) w(t) z(t)>0 .
$$

Lemma 19. Assume that $\left(A_{1}\right)$ holds. Then $\left(A_{2}\right)$ holds if and only if there exists a finite subinterval $\mathscr{I}_{1} \subset \mathscr{I}$ such that one of the following holds:

(1) $\operatorname{rank} \Phi_{\mathscr{I}_{1}}=2 n$;

(2) for some $\lambda \in \mathbb{C}$, every nontrivial solution $y(t)$ of $\left(1_{\lambda}\right)$ satisfies

$$
\sum_{t \in \mathscr{I}_{1}} R(y)^{*}(t) W(t) R(y)(t)>0 .
$$

By Lemma 19, if (52) (or (53)) holds for some $\lambda \in \mathbb{C}$, then it holds for every $\lambda \in \mathbb{C}$. In addition, if $\left(A_{2}\right)$ holds on some finite interval $\mathscr{I}_{1}$, then it holds on $\mathscr{I}_{0}=\left[s_{0}, t_{0}\right]$.

The following is another sufficient and necessary condition for the definiteness condition.

Lemma 20. Assume that $\left(A_{1}\right)$ holds. Then $\left(A_{2}\right)$ holds if and only if for any $\left(y^{\pi}, g^{\pi}\right) \in H(\tau)$, there exists a unique $y \in y^{\pi}$ such that $\tau(y)(t)=W(t) R(g)(t)$ for $t \in \mathscr{I}$.

Remark 21. (1) It can be easily verified that the definiteness condition for $H(\tau)$ holds if and only if that for $H(\delta)$ holds.

(2) In the following of the present paper, we always assume that $\left(A_{2}\right)$ holds. In this case, we can write $\left(y, g^{\pi}\right) \in$ $H(\tau)$ instead of $\left(y^{\pi}, g^{\pi}\right) \in H(\tau)$ in the rest of the present paper.

(3) Denote by $\left(A_{a, 2}\right)$ and $\left(A_{b, 2}\right)$, the definiteness conditions for $\left(1_{\lambda}\right)$ in $\mathscr{I}_{a}$ and $\mathscr{I}_{b}$, and

$$
\mathscr{I}_{a, 0}:=\left[s_{a}^{0}, t_{a}^{0}\right], \quad \mathscr{I}_{b, 0}:=\left[s_{b}^{0}, t_{b}^{0}\right] .
$$


By the corresponding intervals, respectively. It is evident that one of $\left(A_{a, 2}\right)$ and $\left(A_{b, 2}\right)$ implies $\left(A_{2}\right)$.

But $\left(A_{2}\right)$ cannot imply that there exists $c_{0} \in \mathscr{I}$ such that both $\left(A_{a, 2}\right)$ and $\left(A_{b, 2}\right)$ hold.

(4) Several sufficient conditions for the definiteness condition can be given. The reader is referred to [23, Section 4].

For convenience, denote

$$
\begin{gathered}
\mathscr{M}_{\lambda} \\
:=\left\{y \in \mathscr{L}_{W}^{2}(\mathscr{I}): \tau(y)(t)=\lambda W(t) R(y)(t) \text { for } t \in \mathscr{I}\right\}, \\
M_{\lambda}:=\left\{y^{\pi} \in L_{W}^{2}(\mathscr{I}):\left(y^{\pi}, \lambda y^{\pi}\right) \in H(\tau)\right\} .
\end{gathered}
$$

Lemma 22. Assume that $\left(A_{1}\right)$ holds. For any $\lambda \in \mathbb{C}$, $\operatorname{dim} \mathscr{M}_{\lambda}=\operatorname{dim} M_{\lambda}$ if and only if $\left(A_{2}\right)$ holds.

\section{Characterizations of Minimal and Maximal Subspaces and Defect Indices of Minimal Subspaces}

This section is divided into three subsections. In the first subsection, we give all the characterizations of the minimal subspaces generated by $\left(1_{\lambda}\right)$ in $\mathscr{I}, \mathscr{I}_{a}$, and $\mathscr{I}_{b}$. In the second subsection, we study the defect indices of the minimal subspaces. In the third subsection, characterizations of the maximal subspaces are established.

4.1. Characterizations of the Minimal Subspaces. In this subsection, we study characterizations of the minimal subspaces generated by $\left(1_{\lambda}\right)$ in $\mathscr{I}, \mathscr{I}_{a}$, and $\mathscr{I}_{b}$.

The following result is a direct consequence of Theorem 17.

Theorem 23. Assume that $\left(A_{1}\right)$ holds. Then $H_{0}(\tau), H_{b, 0}(\tau)$, and $H_{a, 0}(\tau)$ are closed J-Hermitian subspace in $\left(L_{W}^{2}(\mathscr{I})\right)^{2}$, $\left(L_{W}^{2}\left(\mathscr{I}_{b}\right)\right)^{2}$, and $\left(L_{W}^{2}\left(\mathscr{I}_{a}\right)\right)^{2}$, respectively.

Now, we introduce boundary forms on $\left(L_{W}^{2}(\mathscr{I})\right)^{2}$, $\left(L_{W}^{2}\left(\mathscr{I}_{a}\right)\right)^{2}$, and $\left(L_{W}^{2}\left(\mathscr{I}_{b}\right)\right)^{2}$ by

$$
\begin{gathered}
{[:]:\left(L_{W}^{2}(\mathscr{I})\right)^{2} \times\left(L_{W}^{2}(\mathscr{I})\right)^{2} \rightarrow \mathbb{C},} \\
{\left[\left(x^{\pi}, f^{\pi}\right):\left(y^{\pi}, g^{\pi}\right)\right]=\left\langle f^{\pi}, J y^{\pi}\right\rangle-\left\langle x^{\pi}, J g^{\pi}\right\rangle,} \\
{[:]_{a}:\left(L_{W}^{2}\left(\mathscr{I}_{a}\right)\right)^{2} \times\left(L_{W}^{2}\left(\mathscr{I}_{a}\right)\right)^{2} \longrightarrow \mathbb{C},} \\
{\left[\left(x^{\pi}, f^{\pi}\right):\left(y^{\pi}, g^{\pi}\right)\right]_{a}=\left\langle f^{\pi}, J y^{\pi}\right\rangle_{a}-\left\langle x^{\pi}, J g^{\pi}\right\rangle_{a},} \\
{[:]_{a}:\left(L_{W}^{2}\left(\mathscr{I}_{b}\right)\right)^{2} \times\left(L_{W}^{2}\left(\mathscr{I}_{b}\right)\right)^{2} \longrightarrow \mathbb{C},} \\
{\left[\left(x^{\pi}, f^{\pi}\right):\left(y^{\pi}, g^{\pi}\right)\right]_{b}=\left\langle f^{\pi}, J y^{\pi}\right\rangle_{b}-\left\langle x^{\pi}, J g^{\pi}\right\rangle_{b} .}
\end{gathered}
$$

Lemma 24. Assume that $\left(A_{1}\right)$ holds.

(1) If $\left(A_{2}\right)$ holds, then for any $\left(x, f^{\pi}\right),\left(y, g^{\pi}\right) \in H(\tau)$,

$$
\left[\left(x, f^{\pi}\right):\left(y, g^{\pi}\right)\right]=(x, y)(b+1)-(x, y)(a) .
$$

(2) If $\left(A_{a, 2}\right)$ holds, then for any $\left(x, f^{\pi}\right),\left(y, g^{\pi}\right) \in H_{a}(\tau)$,

$$
\left[\left(x, f^{\pi}\right):\left(y, g^{\pi}\right)\right]_{a}=(x, y)\left(c_{0}\right)-(x, y)(a) .
$$

(3) If $\left(A_{b, 2}\right)$ holds, then for any $\left(x, f^{\pi}\right),\left(y, g^{\pi}\right) \in H_{b}(\tau)$,

$$
\left[\left(x, f^{\pi}\right):\left(y, g^{\pi}\right)\right]_{b}=(x, y)(b+1)-(x, y)\left(c_{0}\right) .
$$

Proof. Since the proofs of (1)-(3) are similar, we only show that assertion (1) holds.

For any $\left(x, f^{\pi}\right),\left(y, g^{\pi}\right) \in H(\tau)$, we have from (2) of Lemma 14 that

$$
\begin{gathered}
\left\langle f^{\pi}, J y^{\pi}\right\rangle-\left\langle x^{\pi}, J g^{\pi}\right\rangle \\
=\sum_{t=s}^{k}\left[R\left(J_{0} y\right)^{*}(t) W(t) R(f)(t)\right. \\
\left.\quad-R\left(J_{0} g\right)^{*}(t) W(t) R(x)(t)\right] \\
=\sum_{t=s}^{k}\left[R\left(J_{0} y\right)^{*}(t) \tau(x)(t)\right. \\
\left.\quad-\left(J_{0} \tau(y)\right)^{*}(t) R(x)(t)\right] \\
=\left.(x, y)(t)\right|_{s} ^{k+1}
\end{gathered}
$$

for any $s<k \in \mathscr{I}$. This yields that $\lim _{t \rightarrow b}(x, y)(t)$ exists and is finite for any $\left(x, f^{\pi}\right),\left(y, g^{\pi}\right) \in H(\tau)$. Similarly, it can be shown that $\lim _{t \rightarrow a}(x, y)(a)$ exists and is finite for any $\left(x, f^{\pi}\right),\left(y, g^{\pi}\right) \in H(\tau)$. Hence, assertion (1) holds. The proof is complete.

Lemma 25. Assume that $\left(A_{1}\right)$ and $\left(A_{2}\right)$ hold. Then for any given finite subset $\mathscr{I}_{1}=[s, k]$ with $\mathscr{I}_{0} \subset \mathscr{I}_{1} \subset \mathscr{I}$ and for any given $\alpha, \beta \in \mathbb{C}^{2 n}$, there exists $f=\{f(t)\}_{t=s}^{k+1} \subset \mathbb{C}^{2 n}$ such that the following boundary value problem:

$$
\begin{gathered}
\tau(x)(t)=W(t) R(f)(t), \quad t \in \mathscr{I}_{1}, \\
x(s)=\alpha, \quad x(k+1)=\beta
\end{gathered}
$$

has a solution $x=\{x(t)\}_{t=s}^{k+1} \subset \mathbb{C}^{2 n}$.

Proof. Set

$$
\langle x, y\rangle^{\prime}:=\sum_{t=s}^{k} R(y)^{*}(t) W(t) R(x)(t), \quad \forall x, y \in l([s, k]) .
$$

Let $\phi_{j}, 1 \leq j \leq 2 n$, be the linearly independent solutions of system $\left(1_{0}\right)$. Then we have

$$
\operatorname{rank}\left(\left\langle\phi_{i}, \phi_{j}\right\rangle^{\prime}\right)_{1 \leq i, j \leq 2 n}=2 n
$$

In fact, the linear algebraic system

$$
\left(\left\langle\phi_{i}, \phi_{j}\right\rangle^{\prime}\right)_{1 \leq i, j \leq 2 n} C=0
$$


where $C=\left(c_{1}, c_{2}, \ldots, c_{2 n}\right)^{T} \in \mathbb{C}^{2 n}$, can be written as

$$
\left\langle\phi_{i}, \sum_{j=1}^{2 n} \bar{c}_{j} \phi_{j}\right\rangle^{\prime}=0, \quad 1 \leq i \leq 2 n,
$$

which yields

$$
\left\langle\sum_{j=1}^{2 n} \bar{c}_{j} \phi_{j}, \sum_{j=1}^{2 n} \bar{c}_{j} \phi_{j}\right\rangle^{\prime}=0
$$

Since $\sum_{j=1}^{2 n} \bar{c}_{j} \phi_{j}$ is a solution of system $\left(1_{0}\right)$, it follows from $\left(A_{2}\right)$ that $\sum_{j=1}^{2 n} \bar{c}_{j} \phi_{j}=0$. Then $C=0$; that is, (65) has only a zero solution. Consequently, (64) holds.

Let $\alpha, \beta$ be any given vectors in $\mathbb{C}^{2 n}$. By (64), the linear algebraic system

$$
\begin{aligned}
& \left(\left\langle\phi_{i}, \phi_{j}\right\rangle^{\prime}\right)_{1 \leq i, j \leq 2 n}^{T} C \\
& \quad=\left(\phi_{1}(k+1), \phi_{2}(k+1), \ldots, \phi_{2 n}(k+1)\right)^{*} \mathscr{J} \beta
\end{aligned}
$$

has a unique solution $C_{1} \in \mathbb{C}^{2 n}$. Set $\psi_{1}=\left(\phi_{1}, \phi_{2}, \ldots, \phi_{2 n}\right) C_{1}$. It follows from (68) that

$$
\left\langle\psi_{1}, \phi_{i}\right\rangle^{\prime}=\phi_{i}^{*}(k+1) \mathscr{J} \beta, \quad 1 \leq i \leq 2 n .
$$

Let $u(t)$ be a solution of the following initial value problem:

$$
\begin{gathered}
\tau(x)(t)=W(t) R\left(\psi_{1}\right)(t), \quad t \in[s, k], \\
x(s)=0 .
\end{gathered}
$$

Since $\tau\left(\phi_{i}\right)(t)=0$ for $t \in \mathscr{I}$ and $1 \leq i \leq 2 n$, we get by (70) and (2) of Lemma 14 that

$$
\begin{aligned}
& \left\langle\psi_{1}, \phi_{i}\right\rangle^{\prime} \\
& \quad=\sum_{t=s}^{k}\left[R\left(\phi_{i}\right)^{*}(t) \tau(u)(t)-\tau\left(\phi_{i}\right)^{*}(t) R(u)(t)\right] \\
& =\left.\left(u, J_{0} \phi_{i}\right)(t)\right|_{s} ^{k+1}=\phi_{i}^{*}(k+1) \mathscr{J} u(k+1), \quad 1 \leq i \leq 2 n .
\end{aligned}
$$

Since $\phi_{1}, \phi_{2}, \ldots, \phi_{2 n}$ are linearly independent in $l(\mathscr{I})$, we get from (69) and (71) that $u(k+1)=\beta$. So, $u(t)$ is a solution of the following boundary value problem:

$$
\begin{gathered}
\tau(x)(t)=W(t) R\left(\psi_{1}\right)(t), \quad t \in[s, k], \\
x(s)=0, \quad x(k+1)=\beta .
\end{gathered}
$$

On the other hand, the linear algebraic system

$$
\left(\left\langle\phi_{i}, \phi_{j}\right\rangle^{\prime}\right)_{1 \leq i, j \leq 2 n}^{T} C=\left(\phi_{1}(s), \phi_{2}(s), \ldots, \phi_{2 n}(s)\right)^{*} J \alpha
$$

has a unique solution $C_{2} \in \mathbb{C}^{2 n}$ by (64). Set $\psi_{2}=\left(\phi_{1}, \phi_{2}, \ldots\right.$, $\left.\phi_{2 n}\right) C_{2}$. Then, by (73)

$$
\left\langle\psi_{2}, \phi_{i}\right\rangle^{\prime}=\phi_{i}^{*}(s) J \alpha, \quad 1 \leq i \leq 2 n .
$$

Let $v(t)$ be a solution of the following initial value problem:

$$
\tau(x)(t)=-W(t) R\left(\psi_{2}\right)(t), \quad x(k+1)=0, \quad t \in[s, k] .
$$

Since $\tau\left(\phi_{i}\right)(t)=0$ for $t \in \mathscr{I}$ and $1 \leq i \leq 2 n$, we get by (2) of Lemma 14 and (75) that

$$
\left\langle\psi_{2}, \phi_{i}\right\rangle^{\prime}=\phi_{i}^{*}(s) J v(s), \quad 1 \leq i \leq 2 n,
$$

which, together with (74), implies that $v(s)=\alpha$. So, $v(t)$ is a solution of the following boundary value problem:

$$
\begin{gathered}
\tau(x)(t)=-W(t) R\left(\psi_{2}\right)(t), \quad t \in[s, k], \\
x(s)=\alpha, \quad x(k+1)=0 .
\end{gathered}
$$

Set $\psi=\psi_{1}-\psi_{2}$ and $\phi=u+v$. Then $\phi$ is a solution of the boundary value problem (62). The proof is complete.

Remark 26. Lemma 25 is called a patch lemma. Based on Lemma 25, any two elements of $H(\tau)\left(H_{b}(\tau), H_{a}(\tau)\right.$, resp.) can be patched up to construct another new element of $H(\tau)$ $\left(H_{b}(\tau), H_{a}(\tau)\right.$, resp.). In particular,

(1) if $\left(A_{b, 2}\right)$ holds, we can take $\mathscr{I}_{1}=\left[c_{0}, t_{b}^{0}\right], \alpha=e_{i}:=$ $(\underbrace{0, \ldots, 0}_{i-1}, 1,0, \ldots, 0)^{T}$, and $\beta=0,1 \leq i \leq 2 n$. Then there exist $\left(z_{b}^{i},\left(h^{\pi}\right)_{b}^{i}\right) \in H_{b}(\tau)$ satisfying

$$
z_{b}^{i}\left(c_{0}\right)=e_{i} ; \quad z_{b}^{i}(t)=0, \quad t \geq t_{b}^{0}+1,1 \leq i \leq 2 n ;
$$

(2) if $\left(A_{a, 2}\right)$ holds, we take $\mathscr{I}_{1}=\left[s_{a}^{0}, c_{0}-1\right], \alpha=0$, and $\beta=e_{i}$. Then there exist $\left(z_{a}^{i},\left(h^{\pi}\right)_{a}^{i}\right) \in H_{a}(\tau)$ satisfying

$$
z_{a}^{i}\left(c_{0}\right)=e_{i} ; \quad z_{a}^{i}(t)=0, \quad t \leq s_{a}^{0}, 1 \leq i \leq 2 n ;
$$

(3) if both $\left(A_{b, 2}\right)$ and $\left(A_{a, 2}\right)$ hold, then there exist $\left(z_{i}, h_{i}^{\pi}\right) \in H(\tau)$ satisfying

$$
\begin{aligned}
& z_{i}\left(c_{0}\right)=e_{i} ; \quad z_{i}(t)=0 \quad \text { for } t \leq s_{a}^{0}, \\
& t \geq t_{b}^{0}+1, \quad 1 \leq i \leq 2 n .
\end{aligned}
$$

The above auxiliary elements $\left(z_{b}^{i},\left(h^{\pi}\right)_{b}^{i}\right),\left(z_{a}^{i},\left(h^{\pi}\right)_{a}^{i}\right)$, and $\left(z_{i}\right.$, $\left.h_{i}^{\pi}\right)(1 \leq i \leq 2 n)$ will be very useful in the sequent discussions.

Theorem 27. Assume that $\left(A_{1}\right)$ holds.

(1) If $\left(A_{2}\right)$ holds, then

$$
\begin{aligned}
& H_{0}(\tau) \\
& =\left\{\left(x, f^{\pi}\right) \in H(\tau):(x, y)(b+1)=(x, y)(a)=0\right. \\
& \quad \forall y \in \operatorname{Dom} H(\tau)\} . \\
& \quad \text { In particular, if } \mathscr{I}=[a, b], \text { then } \\
& H_{00}(\tau) \\
& \quad=\left\{\left(x, f^{\pi}\right) \in H(\tau): x(a)=x(b+1)=0\right\} .
\end{aligned}
$$


(2) If $\left(A_{b, 2}\right)$ holds, then

$$
\begin{aligned}
& H_{b, 0}(\tau) \\
& =\left\{\left(x, f^{\pi}\right) \in H_{b}(\tau): x\left(c_{0}\right)=0,(x, y)(b+1)=0\right. \\
& \left.\quad \forall y \in \operatorname{Dom} H_{b}(\tau)\right\} . \\
& \text { (3) If }\left(A_{a, 2}\right) \text { holds, then } \\
& H_{a, 0}(\tau) \\
& =\left\{\left(x, f^{\pi}\right) \in H_{a}(\tau): x\left(c_{0}\right)=0,(x, y)(a)=0\right. \\
& \left.\quad \forall y \in \operatorname{Dom} H_{a}(\tau)\right\} .
\end{aligned}
$$

Proof. We first show that assertion (1) holds. By Lemmas 8 and 24, and Theorem 17, one has

$$
\begin{gathered}
H_{0}(\tau)=\left\{\left(x, f^{\pi}\right) \in H(\tau):(x, y)(b+1)\right. \\
=(x, y)(a) \\
\forall y \in \operatorname{Dom} H(\tau)\} .
\end{gathered}
$$

For convenience, denote

$$
\begin{gathered}
H^{0}(\tau) \\
=\left\{\left(x, f^{\pi}\right) \in H(\tau):(x, y)(b+1)\right. \\
=(x, y)(a)=0 \\
\forall y \in \operatorname{Dom} H(\tau)\} .
\end{gathered}
$$

Clearly, $H^{0}(\tau) \subset H_{0}(\tau)$. We now show that $H_{0}(\tau) \subset H^{0}(\tau)$. Fix any $\left(x, f^{\pi}\right) \in H_{0}(\tau)$. It follows from (85) that for all $(y$, $\left.g^{\pi}\right) \in H(\tau)$,

$$
(x, y)(b+1)=(x, y)(a) .
$$

For any given $\left(y, g^{\pi}\right) \in H(\tau)$, by Remark 26 there exists $\left(z, h^{\pi}\right) \in H(\tau)$ such that

$$
\begin{gathered}
z(t)=0, \quad t \leq s_{0} \\
z(t)=y(t), \quad t \geq t_{0}+1 .
\end{gathered}
$$

Thus, it follows from $(87)$ that $(x, y)(b+1)=(x, z)(b+1)=$ $(x, z)(a)=0$, and consequently $(x, y)(a)=0$ for all $\left(y, g^{\pi}\right) \in$ $H(\tau)$.

In the case that $\mathscr{I}=[a, b]$, it is clear that

$$
H_{00}(\tau)=\left\{\left(x, f^{\pi}\right) \in H(\tau): x(a)=x(b+1)=0\right\} .
$$

So it remains to show that $H_{0}(\tau)=H_{00}(\tau)$. It suffices to show that $x(a)=x(b+1)=0$ for any $\left(x, f^{\pi}\right) \in H_{0}(\tau)$. Fix any $\left(x, f^{\pi}\right) \in H_{0}(\tau)$, and let $\mathscr{I}_{0}=\mathscr{I}, \alpha=e_{i}, 1 \leq i \leq 2 n$, and $\beta=0$. Then by Lemma 25 , there exist $\left(y_{i}, g_{i}^{\pi}\right) \in H(\tau)$ with $y_{i}(a)=e_{i}$ and $y_{i}(b+1)=0$. Inserting these $y_{i}$ into (87) one has that $x(a)=0$. Similarly, one can show that $x(b+1)=0$. Thus $H_{0}(\tau)=H_{00}(\tau)$. Therefore, assertion (1) has been shown.

With similar arguments, one can show that assertion (2) and (3) hold by using (78) and (79), separately. This completes the proof.
4.2. Defect Indices of Minimal Subspaces. In this subsection, we first give a valued range of the defect indices of $H_{b, 0}(\tau)$ and $H_{a, 0}(\tau)$ and then discuss the relationship among the defect indices of $H_{b, 0}(\tau), H_{a, 0}(\tau)$, and $H_{0}(\tau)$.

For briefness, denote

$$
\begin{gathered}
d:=d\left(H_{0}(\tau)\right), \quad d_{b}:=d\left(H_{b, 0}(\tau)\right), \\
d_{a}:=d\left(H_{a, 0}(\tau)\right) .
\end{gathered}
$$

For any $\lambda \in \mathbb{C}$, let $\mathscr{M}_{b, \lambda}, M_{b, \lambda}, \mathscr{M}_{a, \lambda}$, and $M_{a, \lambda}$ be defined as (56) with $\mathscr{I}$ replaced by $\mathscr{I}_{b}$ and $\mathscr{I}_{a}$, respectively.

The following results are obtained.

Theorem 28. Assume that $\left(A_{1}\right)$ holds.

(1) If $\left(A_{b, 2}\right)$ holds and $\Gamma\left(H_{b, 0}(\tau)\right) \neq \emptyset$, then $d_{b}=\operatorname{dim} \mathscr{M}_{b, \lambda}$ for any $\lambda \in \Gamma\left(H_{b, 0}(\tau)\right)$, and $n \leq d_{b} \leq 2 n$.

(2) If $\left(A_{a, 2}\right)$ holds and $\Gamma\left(H_{a, 0}(\tau)\right) \neq \emptyset$, then $d_{a}=\operatorname{dim} \mathscr{M}_{a, \lambda}$ for any $\lambda \in \Gamma\left(H_{a, 0}(\tau)\right)$, and $n \leq d_{a} \leq 2 n$.

Proof. Since the method of the proofs is the same, we only give the proof of assertion (1).

For any $\lambda \in \Gamma\left(H_{b, 0}(\tau)\right)$, it follows from Lemma 11 and Theorem 18 that

$$
d_{b}=\operatorname{dim} \operatorname{Ker}\left(H_{b}(\delta)-\bar{\lambda}\right)
$$

On the other hand, by using Lemma 5 and Theorems 17 and 18 , one has that

$$
\operatorname{dim} \operatorname{Ker}\left(H_{b}(\tau)-\lambda\right)=\operatorname{dim} \operatorname{Ker}\left(H_{b}(\delta)-\bar{\lambda}\right) .
$$

It is clear that

$$
M_{b, \lambda}=\operatorname{Ker}\left(H_{b}(\tau)-\lambda\right) .
$$

Combining (91)-(93), one has $d_{b}=\operatorname{dim} M_{b, \lambda}$. This, together with Lemma 22, implies that $d_{b}=\operatorname{dim} \mathscr{M}_{b, \lambda}$. In addition, it has been shown in [21] that $n \leq \operatorname{dim} \mathscr{M}_{b, \lambda} \leq 2 n$ for any $\lambda \in \mathbb{C}$. So assertion (1) is true. The proof is complete.

Next, we discuss relationship among defect indices of $H_{0}(\tau), H_{a, 0}(\tau)$, and $H_{b, 0}(\tau)$. For convenience, denote

$$
y_{a}:=\{y(t)\}_{t=a}^{c_{0}}, \quad y_{b}:=\{y(t)\}_{t=c_{0}}^{b+1}
$$

for any $y \in \mathscr{L}_{W}^{2}(\mathscr{I})$. It is evident that $y_{a} \in \mathscr{L}_{W}^{2}\left(\mathscr{I}_{a}\right), y_{b} \in$ $\mathscr{L}_{W}^{2}\left(\mathscr{I}_{b}\right)$, and

$$
\|y\|^{2}=\left\|y_{a}\right\|_{a}^{2}+\left\|y_{b}\right\|_{b}^{2}
$$

On the other hand, for any $y_{a}=\left(u_{a}^{T}, v_{a}^{T}\right)^{T} \in \mathscr{L}_{W}^{2}\left(\mathscr{I}_{a}\right)$ and $y_{b}=\left(u_{b}^{T}, v_{b}^{T}\right)^{T} \in \mathscr{L}_{W}^{2}\left(\mathscr{I}_{b}\right)$, we define $y$ by

$$
y(t)= \begin{cases}y_{a}(t), & t \leq c_{0}-1 \\ \left(u_{a}^{T}\left(c_{0}\right), v_{b}^{T}\left(c_{0}\right)\right)^{T}, & t=c_{0} \\ y_{b}(t), & t \geq c_{0}+1\end{cases}
$$


Then (95) still holds, and consequently $y \in \mathscr{L}_{W}^{2}(\mathscr{I})$. Furthermore, it is clear that

$$
\begin{gathered}
R(y)(t)=R\left(y_{a}\right)(t), \quad t \leq c_{0}-1 \\
R(y)(t)=R\left(y_{b}\right)(t), \quad t \geq c_{0} .
\end{gathered}
$$

The following result can be easily verified. So we omit its proof.

Lemma 29. Assume that $\left(A_{1}\right),\left(A_{a, 2}\right)$, and $\left(A_{b, 2}\right)$ hold.

(1) If $\left(y, g^{\pi}\right) \in H(\tau)$, then $\left(y_{b}, g_{b}^{\pi}\right) \in H_{b}(\tau)$ and $\left(y_{a}, g_{a}^{\pi}\right) \in$ $H_{a}(\tau)$.

(2) If $\left(y_{a}, g_{a}^{\pi}\right) \in H_{a}(\tau)$ and $\left(y_{b}, g_{b}^{\pi}\right) \in H_{b}(\tau)$ with $y_{a}\left(c_{0}\right)=$ $y_{b}\left(c_{0}\right)$, then $\left(y, g^{\pi}\right) \in H(\tau)$.

Let $\widehat{H}_{0}(\tau)$ be the restriction of subspace $H_{0}(\tau)$, defined by

$$
\widehat{H}_{0}(\tau)=\left\{\left(y, g^{\pi}\right) \in H_{0}(\tau): y\left(c_{0}\right)=0\right\} .
$$

Lemma 30. Assume that $\left(A_{1}\right),\left(A_{a, 2}\right)$, and $\left(A_{b, 2}\right)$ hold. Then

(1) $\left(y, g^{\pi}\right) \in \widehat{H}_{0}(\tau)$ if and only if $\left(y_{a}, g_{a}^{\pi}\right) \in H_{a, 0}(\tau)$ and $\left(y_{b}, g_{b}^{\pi}\right) \in H_{b, 0}(\tau)$

(2) $\left(y, g^{\pi}\right) \in\left(\widehat{H}_{0}(\tau)\right)_{J}^{*}$ if and only if $\left(y_{a}, g_{a}^{\pi}\right) \in H_{a}(\tau)$ and $\left(y_{b}, g_{b}^{\pi}\right) \in H_{b}(\tau)$.

Proof. (1) We first consider the necessity. Fix any $\left(y, g^{\pi}\right) \in$ $\widehat{H}_{0}(\tau)$. Let $y_{a}, y_{b}, g_{a}$, and $g_{b}$ be defined as (94). Then $y_{a}\left(c_{0}\right)=$ $y_{b}\left(c_{0}\right)=0$. By (1) of Lemma 29 one has that $\left(y_{a}, g_{a}^{\pi}\right) \in H_{a}(\tau)$, $\left(y_{b}, g_{b}^{\pi}\right) \in H_{b}(\tau)$. In addition, for any $\left(x_{a}, f_{a}^{\pi}\right) \in H_{a}(\tau)$, it follows from Remark 26 that there exits $\left(z, h^{\pi}\right) \in H(\tau)$ with

$$
\begin{aligned}
& z(t)=x_{a}(t), \quad t \leq c_{0}, \\
& z(t)=0, \quad t \geq t_{b}^{0}+1 .
\end{aligned}
$$

So one has

$$
\left(y_{a}, x_{a}\right)(a)=(y, z)(a)=0,
$$

which implies that $\left(y_{a}, g_{a}^{\pi}\right) \in H_{a, 0}(\tau)$ by the arbitrariness of $\left(x_{a}, f_{a}^{\pi}\right) \in H_{a}(\tau)$ and (3) of Theorem 27. With a similar argument, one can show $\left(y_{b}, g_{b}^{\pi}\right) \in H_{b, 0}(\tau)$.

Next, we consider the sufficiency. Fix any $\left(y_{a}, g_{a}^{\pi}\right) \epsilon$ $H_{a, 0}(\tau)$ and $\left(y_{b}, g_{b}^{\pi}\right) \in H_{b, 0}(\tau)$. Let $y$ and $g$ be defined by (96). By (2) and (3) of Theorem 27 one has that $y_{a}\left(c_{0}\right)=y_{b}\left(c_{0}\right)=0$. So $y\left(c_{0}\right)=0$. It follows from (2) of Lemma 29 that $\left(y, g^{\pi}\right) \in$ $H(\tau)$. For any $\left(x, f^{\pi}\right) \in H(\tau)$, it follows from (1) of Lemma 29 that $\left(x_{a}, f_{a}^{\pi}\right) \in H_{a}(\tau)$ and $\left(x_{b}, f_{b}^{\pi}\right) \in H_{b}(\tau)$. Thus one has by (2) and (3) of Theorem 27 that

$$
\begin{gathered}
(y, x)(a)=\left(y_{a}, x_{a}\right)(a)=0, \\
(y, x)(b+1)=\left(y_{b}, x_{b}\right)(b+1)=0 .
\end{gathered}
$$

This implies that $\left(y, g^{\pi}\right) \in H_{0}(\tau)$ by (1) of Theorem 27 , and consequently, $\left(y, g^{\pi}\right) \in \widehat{H}_{0}(\tau)$.

(2) We first consider the necessity. Fix any $\left(y, g^{\pi}\right) \in$ $\left(\widehat{H}_{0}(\tau)\right)_{J}^{*}$. Let $y_{a}, y_{b}, g_{a}$, and $g_{b}$ be defined as (94). Then $\left(y_{a}\right.$, $\left.g_{a}^{\pi}\right) \in\left(L_{W}^{2}\left(\mathscr{I}_{a}\right)\right)^{2}$ and $\left(y_{b}, g_{b}^{\pi}\right) \in\left(L_{W}^{2}\left(\mathscr{I}_{b}\right)\right)^{2}$.
Set $x_{b}(t)=f_{b}(t)=0$ for $t \in \mathscr{I}_{b}^{*}$, where $\mathcal{I}_{b}^{*}$ is defined by (19) with $\mathscr{I}$ replaced by $\mathscr{I}_{b}$. It is clear that $\left(x_{b}, f_{b}^{\pi}\right) \in H_{b, 0}(\tau)$. For any $\left(x_{a}, f_{a}^{\pi}\right) \in H_{a, 0}(\tau)$, let $x$ and $f$ be defined by (96). Then by the above result (1) one has that $\left(x, f^{\pi}\right) \in \widehat{H}_{0}(\tau)$. It follows that

$$
0=\langle f, J y\rangle-\langle x, J g\rangle=\left\langle g_{a}, J y_{a}\right\rangle_{a}-\left\langle x_{a}, J g_{a}\right\rangle_{a} .
$$

By the arbitrariness of $\left(x_{a}, f_{a}^{\pi}\right) \in H_{a, 0}(\tau)$, one has that $\left(y_{a}\right.$, $\left.g_{a}^{\pi}\right) \in\left(H_{a, 0}(\tau)\right)_{J}^{*}=H_{a}(\tau)$ by Theorem 17. With a similar argument one can show $\left(y_{b}, g_{b}^{\pi}\right) \in H_{b}(\tau)$.

Next, we consider the sufficiency. Fix any $\left(y_{a}, g_{a}^{\pi}\right) \in H_{a}(\tau)$ and $\left(y_{b}, g_{b}^{\pi}\right) \in H_{b}(\tau)$. Let $y$ and $g$ be defined by (96). For any $\left(x, f^{\pi}\right) \in \widehat{H}_{0}(\tau)$, it follows from the above result (1) that $\left(x_{a}, f_{a}^{\pi}\right) \in H_{a, 0}(\tau)$ and $\left(x_{b}, f_{b}^{\pi}\right) \in H_{b, 0}(\tau)$. So one has by Theorem 17 that

$$
\begin{aligned}
\left\langle f, J_{0} y\right\rangle & -\left\langle x, J_{0} g\right\rangle \\
= & \left\langle g_{a}, J_{0} y_{a}\right\rangle_{a}-\left\langle x_{a}, J_{0} g_{a}\right\rangle_{a}+\left\langle g_{b}, J_{0} y_{b}\right\rangle_{b} \\
& -\left\langle x_{b}, J_{0} g_{b}\right\rangle_{b}=0 .
\end{aligned}
$$

This yields that $\left(y, g^{\pi}\right) \in\left(\widehat{H}_{0}(\tau)\right)_{J}^{*}$. The whole proof is complete.

Lemma 31. Assume that $\left(A_{1}\right),\left(A_{a, 2}\right)$, and $\left(A_{b, 2}\right)$ hold. Then $\Gamma\left(H_{0}(\tau)\right) \subset \Gamma\left(\widehat{H}_{0}(\tau)\right)$ and $\Gamma\left(H_{b, 0}(\tau)\right) \cap \Gamma\left(H_{a, 0}(\tau)\right)=\Gamma\left(\widehat{H}_{0}(\tau)\right)$.

Proof. The first assertion holds because $\widehat{H}_{0}(\tau) \subset H_{0}(\tau)$, and the second assertion can be proved by (1) of Lemma 30 and (95). The proof is complete.

In the following of the present paper, we assume that

$$
\left(A_{3}\right) \Gamma\left(H_{0}(\tau)\right) \neq \emptyset \text {. }
$$

By Definition 10, one has that if $\Gamma\left(H_{0}(\tau)\right)=\emptyset$, then $\rho\left(H_{0}(\tau)\right)=\emptyset$, and consequently $\sigma\left(H_{0}(\tau)\right)=\mathbb{C}$. We do not consider this case in the present paper.

Theorem 32. Assume that $\left(A_{1}\right),\left(A_{a, 2}\right),\left(A_{b, 2}\right)$, and $\left(A_{3}\right)$ hold. Then

$$
d=d_{a}+d_{b}-2 n
$$

Proof. The proof is divided into two steps.

Step 1. We show that

$$
d\left(\widehat{H}_{0}(\tau)\right)=d_{a}+d_{b}
$$

It follows from $\Gamma\left(H_{0}(\tau)\right) \neq \emptyset$ and Lemma 31 that $\Gamma\left(H_{b, 0}(\tau)\right) \cap \Gamma\left(H_{a, 0}(\tau)\right) \neq \emptyset$. This, together with $\left(A_{a, 2}\right)$, $\left(A_{b, 2}\right)$, and Theorem 28, implies that for any $\lambda \in \Gamma\left(H_{b, 0}(\tau)\right) \cap$ $\Gamma\left(H_{a, 0}(\tau)\right),\left(1_{\lambda}\right)$ has just $d_{b}$ linearly independent solutions $y_{b}^{j} \in \mathscr{L}_{W}^{2}\left(\mathscr{I}_{b}\right), 1 \leq j \leq d_{b}$, and $\left(1_{\lambda}\right)$ has just $d_{a}$ linearly independent solutions $x_{a}^{j} \in \mathscr{L}_{W}^{2}\left(\mathscr{I}_{a}\right), 1 \leq j \leq d_{a}$; that is, $\left(y_{b}^{j}, \lambda\left(y_{b}^{j}\right)^{\pi}\right) \in H_{b}(\tau)$ for $1 \leq j \leq d_{b}$ and $\left(x_{a}^{j}, \lambda\left(x_{a}^{j}\right)^{\pi}\right) \in H_{a}(\tau)$ for $1 \leq j \leq d_{a}$. 
Set $x_{b}^{j}(t)=0$ for $t \in \mathscr{F}_{b}^{*}$. It is clear that $\left(x_{b}^{j}, \lambda\left(x_{b}^{j}\right)^{\pi}\right) \epsilon$ $H_{b}(\tau)$ for $1 \leq j \leq d_{a}$. Let $x_{j}, 1 \leq j \leq d_{a}$, be defined by (96). Then it follows from (2) of Lemma 30 that $\left(x_{j}, \lambda x_{j}^{\pi}\right) \in$ $\left(\widehat{H}_{0}(\tau)\right)_{J}^{*}$. Similarly, set $y_{a}^{j}(t)=0$ for $t \in \mathcal{F}_{a}^{*}$. Then one has $\left(y_{j}, \lambda y_{j}^{\pi}\right) \in\left(\widehat{H}_{0}(\tau)\right)_{J}^{*}$ for $1 \leq j \leq d_{b}$. It is evident that $x_{1}, \ldots$, $x_{d_{a}}, y_{1}, \ldots, y_{d_{b}} \in \operatorname{Dom}\left(\widehat{H}_{0}(\tau)\right)_{J}^{*}$ are linearly independent.

On the other hand, for any $\left(y, \lambda y^{\pi}\right) \in\left(\widehat{H}_{0}(\tau)\right)_{J}^{*}$, it follows from (2) of Lemma 31 that $\left(y_{a}, \lambda y_{a}^{\pi}\right) \in H_{a}(\tau)$ and $\left(y_{b}, \lambda y_{b}^{\pi}\right) \in$ $H_{b}(\tau)$; that is, $y_{a} \in \mathscr{L}_{W}^{2}\left(\mathscr{I}_{a}\right)$ is a solution of $\left(1_{\lambda}\right)$ in $\mathscr{I}_{a}$, and $y_{b} \in \mathscr{L}_{W}^{2}\left(\mathscr{I}_{b}\right)$ is a solution of $\left(1_{\lambda}\right)$ in $\mathscr{I}_{b}$. Therefore, there exist unique $c_{j} \in \mathbb{C}\left(1 \leq j \leq d_{a}\right)$ and $d_{j} \in \mathbb{C}\left(1 \leq j \leq d_{b}\right)$ such that

$$
y_{a}=\sum_{j=1}^{d_{a}} c_{j} x_{a}^{j}, \quad y_{b}=\sum_{j=1}^{d_{b}} d_{j} y_{b}^{j} .
$$

Noting the constructions of $x_{j}$ and $y_{j}$ by (96), one has that

$$
y=\sum_{j=1}^{d_{a}} c_{j} x_{j}+\sum_{j=1}^{d_{b}} d_{j} y_{j} .
$$

This, together with Lemma 11, implies that (105) holds.

Step 2. We show that

$$
d=d\left(\widehat{H}_{0}(\tau)\right)-2 n .
$$

It is evident that $\left(z_{i}, h_{i}^{\pi}\right) \in H_{0}(\tau), 1 \leq i \leq 2 n$, where $\left(z_{i}, h_{i}^{\pi}\right)$ are defined by $(80)$. We claim that

$$
H_{0}(\tau)=\widehat{H}_{0}(\tau)+\operatorname{span}\left\{\left(z_{1}, h_{1}^{\pi}\right),\left(z_{2}, h_{2}^{\pi}\right), \ldots,\left(z_{2 n}, h_{2 n}^{\pi}\right)\right\} .
$$

In fact, for each $\left(y, g^{\pi}\right) \in H_{0}(\tau)$, set $C_{0}=\left(c_{1}, c_{2}, \ldots, c_{2 n}\right)^{T}=$ $y\left(c_{0}\right)$. Let

$$
y_{0}=y-\sum_{i=1}^{2 n} c_{i} z_{i}, \quad g_{0}=g-\sum_{i=1}^{2 n} c_{i} h_{i}
$$

Then

$$
\left(y, g^{\pi}\right)=\left(y_{0}, g_{0}^{\pi}\right)+\sum_{i=1}^{2 n} c_{i}\left(z_{i}, h_{i}^{\pi}\right), \quad\left(y_{0}, g_{0}^{\pi}\right) \in \widehat{H}_{0}(\tau) .
$$

It can be easily verified that this decomposition is unique. Hence, (109) holds.

For any $\lambda \in \Gamma\left(H_{0}(\tau)\right)$, we claim that

$$
\begin{gathered}
\operatorname{Ran}\left(H_{0}(\tau)-\lambda I\right)=\operatorname{Ran}\left(\widehat{H}_{0}(\tau)-\lambda I\right) \\
+\operatorname{span}\left\{h_{1}^{\pi}-\lambda z_{1}^{\pi}, h_{2}^{\pi}-\lambda z_{2}^{\pi}, \ldots,\right. \\
\left.h_{2 n}^{\pi}-\lambda z_{2 n}^{\pi}\right\} .
\end{gathered}
$$

Since (109) holds, it suffices to show that

$$
\begin{gathered}
\operatorname{Ran}\left(\widehat{H}_{0}(\tau)-\lambda I\right) \cap \operatorname{span}\left\{h_{1}^{\pi}-\lambda z_{1}^{\pi}, h_{2}^{\pi}-\lambda z_{2}^{\pi}, \ldots,\right. \\
\left.h_{2 n}^{\pi}-\lambda z_{2 n}^{\pi}\right\}=\{0\} .
\end{gathered}
$$

Suppose that $\left(y, g^{\pi}\right) \in \widehat{H}_{0}(\tau)$ and $c_{1}, c_{2}, \ldots, c_{2 n} \in \mathbb{C}$ satisfy

$$
g^{\pi}-\lambda y^{\pi}+\sum_{j=1}^{2 n} c_{j}\left(h_{j}^{\pi}-\lambda z_{j}^{\pi}\right)=0,
$$

that is,

$$
g^{\pi}+\sum_{j=1}^{2 n} c_{j} h_{j}^{\pi}=\lambda\left(y^{\pi}+\sum_{j=1}^{2 n} c_{j} z_{j}^{\pi}\right) .
$$

Since $\left(y, g^{\pi}\right) \in \widehat{H}_{0}(\tau)$ and $\left(z_{i}, h_{i}^{\pi}\right) \in H_{0}(\tau)$, it follows that $\left(y+\sum_{j=1}^{2 n} c_{j} z_{j}, \lambda\left(y^{\pi}+\sum_{j=1}^{2 n} c_{j} z_{j}^{\pi}\right)\right) \in H_{0}(\tau)$. Since $\lambda \in \Gamma\left(H_{0}(\tau)\right)$, it yields

$$
y+\sum_{j=1}^{2 n} c_{j} z_{j}=0, \quad g^{\pi}+\sum_{j=1}^{2 n} c_{j} h_{j}^{\pi}=0,
$$

which, together with (109), implies that $c_{j}=0$ for $1 \leq j \leq 2 n$, and consequently $g^{\pi}=y^{\pi}=0$. This yields that (113) holds.

Since $H_{0}(\tau)$ and $\widehat{H}_{0}(\tau)$ are closed $J$-Hermitian subspaces, it follows that $\operatorname{Ran}\left(H_{0}(\tau)-\lambda\right)$ and $\operatorname{Ran}\left(\widehat{H}_{0}(\tau)-\lambda\right)$ are closed subspaces in $L_{W}^{2}(\mathscr{I})$, respectively. Hence, there exists a closed subspace $Q$ in $L_{W}^{2}(\mathscr{I})$ such that

$$
\operatorname{Ran}\left(H_{0}(\tau)-\lambda I\right)=\operatorname{Ran}\left(\widehat{H}_{0}(\tau)-\lambda I\right) \oplus Q .
$$

In addition, again by the fact that $\lambda \in \Gamma\left(H_{b, 0}(\tau)\right) \cap \Gamma\left(H_{a, 0}(\tau)\right)$, it follows that $\left\{h_{j}^{\pi}-\lambda z_{j}^{\pi}\right\}_{j=1}^{2 n}$ are linearly independent in $L_{W}^{2}(\mathscr{I})$. It follows from (113) that $\operatorname{dim} Q=2 n$. Consequently,

$$
\begin{aligned}
\operatorname{dim} & \left(\operatorname{Ran}\left(H_{0}(\tau)-\lambda I\right)^{\perp}\right) \\
& =\operatorname{dim}\left(\operatorname{Ran}\left(\widehat{H}_{0}(\tau)-\lambda I\right)^{\perp}\right)-2 n .
\end{aligned}
$$

This yields that (108) holds. It follows from (105) and (108) that (104) holds. The proof is complete.

Remark 33. Theorem 32 (formula (104)) generalizes the classical result for $2 n$th order ordinary differential equations that go back to the classical work by Akhiezer and Glazman [1, Theorem 3 in Appendix 2]. To the case of symmetric Hamiltonian systems, formula (104) was extended in [15].

So it follows from Theorems 28 and 32 that $d=0$ if and only if $d_{a}=d_{b}=n$, and $d=2 n$ if and only if $d_{a}=d_{b}=2 n$. The following definition is obtained.

Definition 34. Assume that $\left(A_{1}\right),\left(A_{2}\right)$, and $\left(A_{b, 2}\right)$ hold. Then $\left(1_{\lambda}\right)$ is said to be in the limit $d_{b}$ case at $t=b$. In the special case that $d_{b}=n,\left(1_{\lambda}\right)$ is said to be in the limit point case (l.p.c.) at $t=b$, and in the other special case that $d_{b}=2 n,\left(1_{\lambda}\right)$ is said to be in the limit circle case (l.c.c.) at $t=b$.

The same definition can be given at $t=a$ provided that $\left(A_{a, 2}\right)$ holds. 
4.3. Characterizations of $H_{a}(\tau)$ and $H_{b}(\tau)$. In this subsection, we characterize the maximal subspaces $H_{a}(\tau)$ and $H_{b}(\tau)$. We first consider characterization of $H_{b}(\tau)$. Assume that $\left(A_{b, 2}\right)$ holds and $\Gamma\left(H_{b, 0}(\tau)\right) \neq \emptyset$. Let $\lambda \in \Gamma\left(H_{b, 0}(\tau)\right)$. It follows from the proof of Lemma 11 that

$$
H_{b}(\tau)=H_{b, 0}(\tau) \dot{+} U_{1} \dot{+} U_{2},
$$

where $U_{1}=\left\{\left(x_{b}^{j}, \lambda\left(x_{b}^{j}\right)^{\pi}\right) \in H_{b}(\tau): 1 \leq j \leq d_{b}\right\}$ and $U_{2}=$ $\left\{\left(y_{b}^{j},\left(g_{b}^{j}\right)^{\pi}\right) \in H_{b}(\tau): 1 \leq j \leq d_{b}\right\}$, and $\left\{\left(y_{b}^{j},\left(g_{b}^{j}\right)^{\pi}\right)\right\}_{j=1}^{d_{b}}$ are linearly independent $\left(\bmod U_{1}\right)$. For convenience, denote

$$
\begin{array}{r}
\chi_{b}^{i}(t):=x_{b}^{i}(t), \quad \chi_{b}^{d_{b}+j}(t):=y_{b}^{j}(t), \\
1 \leq i \leq d_{b}, \quad 1 \leq j \leq d_{b} .
\end{array}
$$

Clearly, $\chi_{b}^{i} \in$ Dom $H_{b}(\tau), 1 \leq i \leq 2 d_{b}$. So $\left(\chi_{b}^{i}, \chi_{b}^{j}\right)(b+1)$ is finite for all $1 \leq i, j \leq 2 d_{b}$. Then the following result can be directly derived from (119) and (120).

Lemma 35. Assume that $\left(A_{1}\right)$ and $\left(A_{b, 2}\right)$ hold, and $\Gamma\left(H_{b, 0}(\tau)\right) \neq \emptyset$. Then every $y \in$ Dom $H_{b}(\tau)$ can be expressed as

$$
y=y_{b}^{0}+\sum_{i=1}^{2 d_{b}} a_{i} \chi_{b}^{i}
$$

where $y_{b}^{0} \in \operatorname{Dom} H_{b, 0}(\tau)$ and $a_{i} \in \mathbb{C}$.

By Lemma 35, $z_{b}^{i}$, defined by (78), can be uniquely expressed as

$$
z_{b}^{i}=y_{b}^{i, 0}+\sum_{j=1}^{2 d_{b}} a_{i j} \chi_{b}^{j}, \quad 1 \leq i \leq 2 n
$$

where $y_{b}^{i, 0} \in \operatorname{Dom} H_{b, 0}(\tau)$ and $a_{i j} \in \mathbb{C}$. Denote

$$
E:=\left(a_{i j}\right)_{2 n \times 2 d_{b}}, \quad F_{1}:=\left(\left(\chi_{b}^{i}, \chi_{b}^{j}\right)(b+1)\right)_{1 \leq i, j \leq 2 d_{b}} .
$$

Lemma 36. Assume that $\left(A_{1}\right)$ and $\left(A_{b, 2}\right)$ hold, and $\Gamma\left(H_{b, 0}(\tau)\right) \neq \emptyset$. Then rank $E=2 n$ and $\operatorname{rank} F_{1}=2 d_{b}-2 n$. Furthermore, we can rearrange the order of $\chi_{b}^{1}, \chi_{b}^{2}, \ldots, \chi_{b}^{d_{b}}$ such that

$$
\operatorname{rank}\left(\left(\chi_{b}^{i}, \chi_{b}^{j}\right)(b+1)\right)_{1 \leq i \leq 2 d_{b}-2 n, 1 \leq j \leq d_{b}}=2 d_{b}-2 n .
$$

Proof. It follows from (122) that

$$
\begin{aligned}
& \left(z_{b}^{1}, z_{b}^{2}, \ldots, z_{b}^{2 n}\right) \\
& \quad=\left(y_{b}^{1,0}, y_{b}^{2,0}, \ldots, y_{b}^{2 n, 0}\right)+\left(\chi_{b}^{1}, \chi_{b}^{2}, \ldots, \chi_{b}^{2 d_{b}}\right) E^{T},
\end{aligned}
$$

which, together with (78), implies that

$$
I_{2 n}=\left(\chi_{b}^{1}\left(c_{0}\right), \chi_{b}^{2}\left(c_{0}\right), \ldots, \chi_{b}^{2 d_{b}}\left(c_{0}\right)\right) E^{T} .
$$

By Lemma 12, one has rank $E=2 n$.
On the other hand, it follows from (122) that

$$
\left(z_{b}^{i}, \chi_{b}^{s}\right)(b+1)=\left(y_{b}^{i, 0}, \chi_{b}^{s}\right)(b+1)+\sum_{j=1}^{2 d_{b}} a_{i j}\left(\chi_{j}^{b}, \chi_{b}^{s}\right)(b+1),
$$

for $1 \leq i \leq 2 n, 1 \leq s \leq 2 d_{b}$, which, together with (78) and (2) of Theorem 27, implies that

$$
E F_{1}=0
$$

Noting that rank $E=2 n$, one has

$$
\operatorname{rank} F_{1} \leq 2 d_{b}-2 n
$$

We now want to show rank $F_{1} \geq 2 d_{b}-2 n$. By (3) of Lemma 14 one has

$$
F_{2}:=\left(\left(\chi_{b}^{i}, \chi_{b}^{j}\right)(b+1)\right)_{1 \leq i \leq d_{b}, 1 \leq j \leq d_{b}}=\left(X_{0}^{T} \mathscr{J} X_{0}\right)^{T},
$$

where $X_{0}:=\left(x_{b}^{1}\left(c_{0}\right), x_{b}^{2}\left(c_{0}\right), \ldots, x_{b}^{d_{b}}\left(c_{0}\right)\right)$. Since rank $X_{0}=d_{b}$, we have that

$$
\operatorname{rank} F_{1} \geq \operatorname{rank} F_{2} \geq 2 d_{b}-2 n,
$$

which, together with (129), yields that

$$
\operatorname{rank} F_{1}=\operatorname{rank} F_{2}=2 d_{b}-2 n \text {. }
$$

Because rank $F_{2}=2 d_{b}-2 n \leq d_{b}$, one can rearrange the order of $\chi_{b}^{1}, \chi_{b}^{2}, \ldots, \chi_{b}^{d_{b}}$ such that the first $2 d_{b}-2 n$ rows of $F_{2}$ are linearly independent; that is, (124) holds. The proof is complete.

Without loss of generality, we assume that (124) holds in the rest of this paper. Now, we can give a characterization of $H_{b}(\tau)$.

Theorem 37. Assume that $\left(A_{1}\right)$ and $\left(A_{b, 2}\right)$ hold, $\lambda \in$ $\Gamma\left(H_{b, 0}(\tau)\right) \neq \emptyset$, and $\chi_{b}^{1}, \chi_{b}^{2}, \ldots, \chi_{b}^{2 d_{b}-2 n}$ are linearly independent solutions of $\left(1_{\lambda}\right)$ in $\mathscr{L}_{w}^{2}\left(\mathscr{I}_{b}\right)$ such that (124) holds. Then

$$
G_{b}:=\left(\left(\chi_{b}^{i}, \chi_{b}^{j}\right)(b+1)\right)_{1 \leq i, j \leq 2 d_{b}-2 n}
$$

is invertible, and any $y \in \operatorname{Dom} H_{b}(\tau)$ can be uniquely expressed as

$$
y=y_{b}^{0}+\sum_{i=1}^{2 n} c_{i} z_{b}^{i}+\sum_{j=1}^{2 d_{b}-2 n} d_{j} \chi_{b}^{j}
$$

where $y_{b}^{0} \in \operatorname{Dom} H_{b, 0}(\tau), z_{b}^{i}$ are defined by (78), and $c_{i}, d_{j} \in$ $\mathbb{C}$.

Proof. Let $E=\left(E_{1}, E_{2}\right)$, where $E_{1}$ and $E_{2}$ are $2 n \times\left(2 d_{b}-2 n\right)$ and $2 n \times 2 n$ matrices, respectively. It follows from (124) that there exists an invertible matrix $L$ such that

$$
F_{1} L=\left(\begin{array}{cc}
I_{2 d_{b}-2 n} & 0 \\
F_{3} & F_{4}
\end{array}\right) .
$$


So, it follows from (128) that $E_{1}+E_{2} F_{3}=0$, which is equivalent to $E_{1}=-E_{2} F_{3}$. Since rank $E=2 n$, it follows that $E_{2}$ is invertible. Multiplying (125) by $\left(E_{2}^{-1}\right)^{T}$ from the right-hand side, we get

$$
\begin{aligned}
\left(z_{b}^{1}, z_{b}^{2}, \ldots, z_{b}^{2 n}\right)\left(E_{2}^{-1}\right)^{T}= & \left(y_{b}^{1,0}, y_{b}^{2,0}, \ldots, y_{b}^{2 n, 0}\right)\left(E_{2}^{-1}\right)^{T} \\
& +\left(\chi_{b}^{1}, \chi_{b}^{2}, \ldots, \chi_{b}^{2 d_{b}-2 n}\right)\left(E_{2}^{-1} E_{1}\right)^{T} \\
& +\left(\chi_{b}^{2 d_{b}-2 n+1}, \ldots, \chi_{b}^{2 d_{b}}\right)
\end{aligned}
$$

This implies that each of $\chi_{b}^{2 d_{b}-2 n+1}, \ldots, \chi_{b}^{2 d_{b}}$ can be uniquely expressed as a linear combination of $\chi_{b}^{1}, \chi_{b}^{2}, \ldots, \chi_{b}^{2 d_{b}-2 n}, y_{b}^{1,0}$, $y_{b}^{2,0}, \ldots, y_{b}^{2 n, 0}$, and $z_{b}^{1}, z_{b}^{2}, \ldots, z_{b}^{2 n}$. Therefore, (134) follows from Lemma 35.

Since $\chi_{b}^{j} \in \operatorname{Dom} H_{b}(\tau)$ for $1 \leq j \leq d_{b}, \chi_{b}^{j}$ can be uniquely expressed as

$$
\chi_{b}^{j}=y_{b}^{j, 0}+\sum_{l=1}^{2 n} b_{j l} z_{b}^{l}+\sum_{s=1}^{2 d_{b}-2 n} c_{j s} \chi_{b}^{s}, \quad 1 \leq j \leq d_{b},
$$

where $y_{b}^{j, 0} \in \operatorname{Dom} H_{b, 0}(\tau)$, and $b_{j l}, c_{j s} \in \mathbb{C}$. This, together with (78) and (2) of Theorem 27, implies that for $1 \leq i \leq$ $2 d_{b}-2 n, 1 \leq j \leq d_{b}$

$$
\left(\chi_{b}^{i}, \chi_{b}^{j}\right)(b+1)=\sum_{s=1}^{2 d_{b}-2 n} c_{j s}\left(\chi_{b}^{i}, \chi_{s}^{b}\right)(b+1),
$$

that is,

$$
\left(\chi_{b}^{i}, \chi_{b}^{j}\right)(b+1)_{1 \leq i \leq 2 d_{b}-2 n, 1 \leq j \leq d_{b}}=G_{b}\left(c_{i j}\right)_{1 \leq i \leq d_{b}, 1 \leq j \leq 2 d_{b}-2 n}^{T} .
$$

Therefore, $G_{b}$ is invertible from (124). This completes the proof.

With a similar argument, one can obtain the following characterization of $H_{a}(\tau)$.

Theorem 38. Assume that $\left(A_{1}\right)$ and $\left(A_{a, 2}\right)$ hold, $\Gamma\left(H_{a, 0}(\tau)\right) \neq$ $\emptyset$. Then, for some $\lambda \in \Gamma\left(H_{a, 0}(\tau)\right)$, system $\left(1_{\lambda}\right)$ has $2 d_{a}-2 n$ linearly independent solutions $\chi_{a}^{1}, \ldots, \chi_{a}^{2 d_{a}-2 n}$ in $\mathscr{L}_{W}^{2}\left(\mathscr{I}_{a}\right)$ such that $G_{a}$ is invertible, where

$$
G_{a}:=\left(\left(\chi_{a}^{i}, \chi_{a}^{j}\right)(a)\right)_{1 \leq i, j \leq 2 d_{a}-2 n}
$$

and each $y \in \operatorname{Dom} H_{a}(\tau)$ can be uniquely expressed as

$$
y=y_{a}^{0}+\sum_{i=1}^{2 n} c_{i} z_{a}^{i}+\sum_{j=1}^{2 d_{a}-2 n} d_{j} \chi_{a}^{j}
$$

where $y_{a}^{0} \in \operatorname{Dom} H_{a, 0}(\tau), c_{i}, d_{j} \in \mathbb{C}$, and $z_{a}^{i}$ are defined as (79).

\section{Characterizations of $J$-SSEs of $H_{0}(\tau)$}

In this section, we give a complete characterization of all the $J$-SSEs of minimal subspace $H_{0}(\tau)$ in terms of the square summable solutions of system $\left(1_{\lambda}\right)$. As a consequence, characterizations of all the $J$-self-adjoint subspace extensions are obtained in the two special cases: the limit point and limit circle cases. The following discussion is divided into two parts based on the form of $\mathscr{I}$.

5.1. Both the Endpoints Are Infinite. Let $\mathscr{I}=(-\infty,+\infty)$, and assume that $\left(A_{1}\right),\left(A_{a, 2}\right)$, and $\left(A_{b, 2}\right)$ hold, and $\Gamma\left(H_{0}(\tau)\right) \neq \emptyset$ in this subsection. It follows from Theorem 32 that

$$
d_{a}+d_{b}-2 n=d
$$

In addition, for some $\lambda \in \Gamma\left(H_{0}(\tau)\right.$, let $\chi_{b}^{1}, \ldots, \chi_{b}^{2 d_{b}-2 n}$ given in Theorems 37 and $\chi_{a}^{1}, \ldots, \chi_{a}^{2 d_{a}-2 n}$ be given in Theorems 38 .

Theorem 39. Assume that $\left(A_{1}\right),\left(A_{a, 2}\right),\left(A_{b, 2}\right)$, and $\left(A_{3}\right)$ hold. Then a subspace $T \subset\left(L_{W}^{2}(\mathscr{I})\right)^{2}$ is a J-SSE of $H_{0}(\tau)$ if and only if there exist two matrices $M_{d \times\left(2 d_{a}-2 n\right)}$ and $N_{d \times\left(2 d_{b}-2 n\right)}$ such that

$$
\begin{aligned}
& \text { (1) } \operatorname{rank}(M, N)=d \text {, } \\
& \text { (2) } N G_{b} N^{T}-M G_{a} M^{T}=0 \text {, and } \\
& T=\left\{\begin{array}{c}
\left(y, \chi_{a}^{1}\right)(-\infty) \\
\left(y, g^{\pi}\right) \in H(\tau): M\left(\begin{array}{c}
\left(y, \chi_{b}^{1}\right)(+\infty) \\
\left(y, \chi_{a}^{2 d_{a}-2 n}\right)(-\infty)
\end{array}\right) \\
\left.-N\left(\begin{array}{c}
\left(y, \chi_{b}^{2 d_{b}-2 n}\right)(+\infty)
\end{array}\right)=0\right\},
\end{array}\right.
\end{aligned}
$$

where $G_{b}$ and $G_{a}$ are the same as those in Theorems 37 and 38 . Proof. We first show the sufficiency.

Suppose that there exist two matrices $M_{d \times\left(2 d_{a}-2 n\right)}$ and $N_{d \times\left(2 d_{b}-2 n\right)}$ such that conditions (1) and (2) hold and $T$ is defined by (143). We now prove that $T$ is a $J$-self-adjoint subspace extension of $H_{0}(\tau)$ by Lemma 9 .

Denote

$$
M=\left(m_{i j}\right)_{d \times\left(2 d_{a}-2 n\right)}, \quad N=\left(n_{i j}\right)_{d \times\left(2 d_{b}-2 n\right)},
$$

and set

$$
w_{a}^{i}:=\sum_{j=1}^{2 d_{a}-2 n} m_{i j} \chi_{a}^{j}, \quad w_{b}^{i}:=\sum_{j=1}^{2 d_{b}-2 n} n_{i j} \chi_{b}^{j}, \quad 1 \leq i \leq d .
$$


Clearly, $w_{a}^{i} \in \operatorname{Dom} H_{a}(\tau)$ and $w_{b}^{i} \in \operatorname{Dom} H_{b}(\tau)$ for $1 \leq i \leq d$. By Remark 26, there exist $\beta_{i}:=\left(\omega_{i}, \rho_{i}^{\pi}\right) \in H(\tau)(1 \leq i \leq d)$ such that

$$
\begin{gathered}
\omega_{i}(t)=w_{a}^{i}(t), \quad t \leq s_{a}^{0}, \\
\omega_{i}(t)=w_{b}^{i}(t), \quad t \geq t_{b}^{0}+1,
\end{gathered}
$$

where $s_{a}^{0}$ and $t_{b}^{0}$ are specified by $\left(A_{a, 2}\right)$ and $\left(A_{b, 2}\right)$, respectively.

Since $H(\tau)$ and $H_{0}(\tau)$ are liner subspaces, $\beta_{1}, \beta_{2}, \ldots, \beta_{d}$ are linearly independent in $H(\tau)$ (modulo $H_{0}(\tau)$ ) if and only if $\omega_{1}, \omega_{2}, \ldots, \omega_{d}$ are linearly independent in Dom $H(\tau)$ (modulo Dom $H_{0}(\tau)$ ). So it suffices to show that $\omega_{1}, \omega_{2}, \ldots$, $\omega_{d}$ are linearly independent in Dom $H(\tau)$ (modulo Dom $\left.H_{0}(\tau)\right)$. Suppose that there exists $C=\left(c_{1}, c_{2}, \ldots, c_{d}\right) \in$ $\mathbb{C}^{d}$ such that

$$
\omega=\sum_{j=1}^{d} c_{j} \omega_{j} \in \operatorname{Dom} H_{0}(\tau)
$$

It follows from (145), (146), and (2) and (3) of Theorem 27 that

$$
\begin{aligned}
& 0=\left(\left(\omega, \chi_{a}^{1}\right)(-\infty), \ldots,\left(\omega, \chi_{a}^{2 d_{a}-2 n}\right)(-\infty)\right)=C M G_{a}, \\
& 0=\left(\left(\omega, \chi_{b}^{1}\right)(+\infty), \ldots,\left(\omega, \chi_{b}^{2 d-2 n}\right)(+\infty)\right)=C N G_{b} .
\end{aligned}
$$

Since $G_{b}$ and $G_{a}$ are invertible, we get from (148) that $C M=$ $C N=0$. Then $C=0$ by condition (1). So, $\omega_{1}, \omega_{2}, \ldots, \omega_{d}$ are linearly independent in Dom $H(\tau)$ (modulo Dom $H_{0}(\tau)$ ), and consequently $\beta_{1}, \beta_{2}, \ldots, \beta_{d}$ are linearly independent in $H(\tau)$ (modulo $H_{0}(\tau)$ ).

Next, we show that $\left[\beta_{i}: \beta_{j}\right]=0$ for $1 \leq i, j \leq d$. It follows from (145) and (146) that

$$
\begin{aligned}
& \left(\left(\omega_{i}, \omega_{j}\right)(-\infty)\right)_{1 \leq i, j \leq d}=M G_{a} M^{T}, \\
& \left(\left(\omega_{i}, \omega_{j}\right)(+\infty)\right)_{1 \leq i, j \leq d}=N G_{b} N^{T},
\end{aligned}
$$

which, together with Lemma 24 and condition (2), implies that

$$
\left(\left[\beta_{i}: \beta_{j}\right]\right)_{1 \leq i, j \leq d}=N G_{b} N^{T}-M G_{a} M^{T}=0 .
$$

Consequently, $\left[\beta_{i}: \beta_{j}\right]=0$ for $1 \leq i, j \leq d$. Therefore, $\left\{\beta_{j}\right\}_{j=1}^{d}$ satisfy the conditions (1) and (2) of Lemma 9.
Note that for each $y \in \operatorname{Dom} H(\tau)$, it follows that

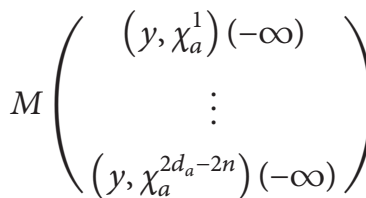

$$
=\left(\begin{array}{c}
\left(y, \sum_{j=1}^{2 d_{a}-2 n} m_{1 j} \chi_{a}^{j}\right)(-\infty) \\
\vdots \\
\left(y, \sum_{j=1}^{2 d_{a}-2 n} m_{d j} \chi_{a}^{j}\right)(-\infty)
\end{array}\right)
$$$$
=\left(\begin{array}{c}
\left(y, \omega_{1}\right)(-\infty) \\
\vdots \\
\left(y, \omega_{d}\right)(-\infty)
\end{array}\right) \text {, }
$$$$
N\left(\begin{array}{c}
\left(y, \chi_{b}^{1}\right)(+\infty) \\
\vdots \\
\left(y, \chi_{b}^{2 d-2 n}\right)(+\infty)
\end{array}\right)
$$

$$
\begin{aligned}
& =\left(\begin{array}{c}
\left(y, \sum_{j=1}^{2 d-2 n} n_{1 j} \chi_{b}^{j}\right)(+\infty) \\
\vdots \\
\left(y, \sum_{j=1}^{2 d-2 n} n_{d j} \chi_{b}^{j}\right)(+\infty)
\end{array}\right) \\
& =\left(\begin{array}{c}
\left(y, \omega_{1}\right)(+\infty) \\
\vdots \\
\left(y, \omega_{d}\right)(+\infty)
\end{array}\right) \text {, }
\end{aligned}
$$

where (145) and (146) have been used. Therefore, it follows from Lemma 24 that $T$ can be expressed as

$$
T=\left\{\left(y, g^{\pi}\right) \in H(\tau):\left[\left(y, g^{\pi}\right): \beta_{j}\right]=0,1 \leq j \leq d\right\} .
$$

Hence, $T$ is a $J$-SSE of $H_{0}(\tau)$ by Lemma 9. The sufficiency is proved.

We now show the necessity. Suppose that $T$ is a $J$-SSE of $H_{0}(\tau)$. By Lemma 9 and Theorem 17, there exists a set of $\left\{\beta_{j}\right\}_{j=1}^{d} \subset H(\tau)$ such that (152) holds. Write $\beta_{j}=\left(\omega_{j}, \rho_{j}^{\pi}\right)$. Then $\omega_{a}^{j} \in \operatorname{Dom} H_{a}(\tau)$ and $\omega_{b}^{j} \in \operatorname{Dom} H_{b}(\tau)$ for $1 \leq j \leq d$. 
By Theorems 37 and 38, each $\omega_{a}^{j}$ and $\omega_{b}^{j}$ can be uniquely expressed as

$$
\begin{gathered}
\omega_{a}^{j}=y_{a}^{j, 0}+\sum_{i=1}^{2 n} c_{i j} z_{a}^{i}+\sum_{s=1}^{2 d_{a}-2 n} m_{j s} \chi_{a}^{s}, \\
\omega_{b}^{j}=y_{b}^{j, 0}+\sum_{i=1}^{2 n} d_{i j} z_{b}^{i}+\sum_{s=1}^{2 d_{b}-2 n} n_{j s} \chi_{b}^{s}, \quad 1 \leq j \leq d,
\end{gathered}
$$

where $y_{b}^{j, 0} \in \operatorname{Dom} H_{b, 0}(\tau), y_{a}^{j, 0} \in \operatorname{Dom} H_{a, 0}(\tau)$, and $d_{i j}, c_{i j}$, $n_{j s}, m_{j s} \in \mathbb{C}$. Set

$$
M=\left(m_{j s}\right)_{d \times\left(2 d_{a}-2 n\right)^{\prime}}, \quad N=\left(n_{j s}\right)_{d \times\left(2 d_{b}-2 n\right)^{\prime}} .
$$

First, we want to show that $M$ and $N$ satisfy condition (1). Otherwise, suppose that $\operatorname{rank}(M, N)<d$. Then there exists $C=\left(c_{1}, c_{2}, \ldots, c_{d}\right) \in \mathbb{C}^{d}$ with $C \neq 0$ such that

$$
C(M, N)=0 .
$$

Then $C M=0$ and $C N=0$. Let $\omega=\sum_{j=1}^{d} c_{j} \omega_{j}$. We then have

$$
\begin{aligned}
& \left(\left(\omega, \chi_{a}^{1}\right)(-\infty), \ldots,\left(\omega, \chi_{a}^{2 d_{a}-2 n}\right)(-\infty)\right)=C M G_{a}=0 \\
& \left(\left(\omega, \chi_{b}^{1}\right)(+\infty), \ldots,\left(\omega, \chi_{b}^{2 d-2 n}\right)(+\infty)\right)=C N G_{b}=0 .
\end{aligned}
$$

For each $y \in \operatorname{Dom} H(\tau), y_{b}$ can be uniquely expressed as (134) by Theorem 37 and $y_{a}$ can be uniquely expressed as (141) by Theorem 38. So it follows from (156), (78), (79), and (2) and (3) of Theorem 27 that for any $y \in \operatorname{Dom} H(\tau),(\omega, y)(+\infty)=$ $(\omega, y)(-\infty)=0$, which yields that $\omega \in$ Dom $H_{0}(\tau)$ by (1) of Theorem 27. Then $\omega_{1}, \omega_{2}, \ldots, \omega_{d}$ are linearly dependent in Dom $H(\tau)$ (modulo Dom $H_{0}(\tau)$ ), and consequently $\beta_{1}, \beta_{2}, \ldots, \beta_{d}$ are linearly dependent in $H(\tau)$ (modulo $H_{0}(\tau)$ ). This is a contradiction. Hence, $\operatorname{rank}(M, N)=d$.

Next, we prove that $M$ and $N$ satisfy condition (2). It can be easily verified that

$$
\begin{aligned}
& \left(\left(\omega_{i}, \omega_{j}\right)(-\infty)\right)_{1 \leq i, j \leq d}=M G_{a} M^{T}, \\
& \left(\left(\omega_{i}, \omega_{j}\right)(+\infty)\right)_{1 \leq i, j \leq d}=N G_{b} N^{T} .
\end{aligned}
$$

Hence, by Lemma 14 and $\left[\beta_{i}: \beta_{j}\right]=0$ for $1 \leq i, j \leq d, M$ and $N$ satisfy condition (2).
In addition, it follows from (78), (79), (153), and (2) and (3) of Theorem 27 that

$$
\begin{aligned}
& \left(\begin{array}{c}
\left(y, \omega_{1}\right)(-\infty) \\
\vdots \\
\left(y, \omega_{d}\right)(-\infty)
\end{array}\right) \\
& =\left(\begin{array}{c}
\left(y, \sum_{j=1}^{2 d_{a}-2 n} m_{1 j} \chi_{a}^{j}\right)(-\infty) \\
\vdots \\
\left(y, \sum_{j=1}^{2 d_{a}-2 n} m_{d j} \chi_{a}^{j}\right)(-\infty)
\end{array}\right) \\
& =M\left(\begin{array}{c}
\left(y, \chi_{a}^{1}\right)(-\infty) \\
\vdots \\
\left(y, \chi_{a}^{2 d_{a}-2 n}\right)(-\infty)
\end{array}\right) \text {, } \\
& \left(\begin{array}{c}
\left(y, \omega_{1}\right)(+\infty) \\
\vdots \\
\left(y, \omega_{d}\right)(+\infty)
\end{array}\right) \\
& =\left(\begin{array}{c}
\left(y, \sum_{j=1}^{2 d-2 n} n_{1 j} \chi_{b}^{j}\right)(+\infty) \\
\vdots \\
\left(y, \sum_{j=1}^{2 d-2 n} n_{d j} \chi_{b}^{j}\right)(+\infty)
\end{array}\right) \\
& =N\left(\begin{array}{c}
\left(y, \chi_{b}^{1}\right)(+\infty) \\
\vdots \\
\left(y, \chi_{b}^{2 d-2 n}\right)(+\infty)
\end{array}\right) \text {. }
\end{aligned}
$$

Hence, $T$ in (152) can be expressed as (143). The necessity is proved.

The entire proof is complete.

To end this subsection, we give characterizations of $J$ SSEs of $H_{0}(\tau)$ in four special cases of defect indices: $d_{a}=d_{b}=$ $n ; d_{a}=n, d_{b}=2 n ; d_{a}=2 n, d_{b}=n ; d_{a}=d_{b}=2 n$.

In the case that $d_{a}=d_{b}=n$, that is, $d=0$ by Theorem 32, the following result is derived from Lemma 11 and Theorem 17.

Theorem 40. Assume that $\left(A_{1}\right),\left(A_{a, 2}\right),\left(A_{b, 2}\right)$, and $\left(A_{3}\right)$ hold. If $d=0$, then $H_{0}(\tau)$ is a J-self-adjoint subspace. 
In the case that $d_{a}=n, d_{b}=2 n$, it follows from Theorem 32 that $d=n$. Let $\chi_{b}^{i},(1 \leq i \leq 2 n)$, be $2 n$ linearly independent solutions in $\mathscr{L}_{W}^{2}\left(\mathscr{I}_{b}\right)$ of $\left(1_{\lambda}\right)$ satisfying $\left(\chi_{b}^{1}\left(c_{0}\right), \chi_{b}^{2}\left(c_{0}\right), \ldots, \chi_{b}^{2 n}\left(c_{0}\right)\right)=I_{2 n}$. Then, by (3) of Lemma 14 one has that

$$
G_{b}=\left(\left(\chi_{b}^{i}, \chi_{b}^{j}\right)\left(c_{0}\right)\right)_{1 \leq i, j \leq 2 n}=\left(J_{0} Y\right)^{*}\left(c_{0}\right) \mathscr{J} Y\left(c_{0}\right)=\mathscr{J} .
$$

The following result can be directly derived from Theorem 39 .

Theorem 41. Assume that $\left(A_{1}\right),\left(A_{2}\right),\left(A_{a, 2}\right),\left(A_{b, 2}\right)$, and $\left(A_{3}\right)$ hold. If $\left(1_{\lambda}\right)$ is in l.p.c. at $t=-\infty$ and in l.c.c. at $t=+\infty$. Let $\chi_{b}^{i}(1 \leq i \leq 2 n)$ be $2 n$ linearly independent solutions in $\mathscr{L}_{W}^{2}\left(\mathscr{I}_{b}\right)$ of $\left(1_{\lambda}\right)$ satisfying $\left(\chi_{1}^{b}\left(c_{0}\right), \chi_{2}^{b}\left(c_{0}\right), \ldots, \chi_{2 n}^{b}\left(c_{0}\right)\right)=I_{2 n}$. Then a subspace $T \subset\left(L_{W}^{2}(\mathscr{I})\right)^{2}$ is a J-SSE of $H_{0}(\tau)$ if and only if there exists a matrix $N_{n \times 2 n}$ such that

$$
\begin{gathered}
\operatorname{rank} N=n, \quad N \mathscr{g} N^{T}=0, \\
T=\left\{\left(y, g^{\pi}\right) \in H(\tau): N\left(\begin{array}{c}
\left(y, \chi_{b}^{1}\right)(+\infty) \\
\vdots \\
\left(y, \chi_{b}^{2 n}\right)(+\infty)
\end{array}\right)=0\right\} .
\end{gathered}
$$

In the case that $d_{a}=2 n, d_{b}=n$, a similar result can be easily given. So we omit the details in this case.

In the case that $d_{a}=d_{b}=2 n$, it follows from Theorem 32 that $d=2 n$. Let $\chi_{i}(1 \leq i \leq 2 n)$ be $2 n$ linearly independent solutions in $\mathscr{L}_{W}^{2}(\mathscr{I})$ of $\left(1_{\lambda}\right)$ satisfying

$$
\left(\chi_{1}\left(c_{0}\right), \chi_{2}\left(c_{0}\right), \ldots, \chi_{2 n}\left(c_{0}\right)\right)=I_{2 n} .
$$

Then, by Lemma $14, G_{b}$ and $G_{a}$, defined by (133) and (140), satisfy

$$
G_{a}=G_{b}=\mathscr{J} .
$$

The following result is a direct consequence of Theorem 39.

Theorem 42. Assume that $\left(A_{1}\right),\left(A_{a, 2}\right),\left(A_{b, 2}\right)$, and $\left(A_{3}\right)$ hold. If $\left(1_{\lambda}\right)$ is in l.c.c. at both $t=+\infty$ and $t=-\infty$, then for any given $\lambda \in \Gamma\left(H_{0}(\tau)\right)$, let $\chi_{i}(1 \leq i \leq 2 n)$ be $2 n$ linearly independent solutions in $\mathscr{L}_{W}^{2}(\mathscr{I})$ of $\left(1_{\lambda}\right)$ satisfying $\left(\chi_{1}\left(c_{0}\right), \chi_{2}\left(c_{0}\right), \ldots, \phi_{2 n}\left(c_{0}\right)\right)=I_{2 n}$. Then a subspace $T \mathrm{C}$ $\left(L_{W}^{2}(\mathscr{I})\right)^{2}$ is a J-SSE of $H_{0}(\tau)$ if and only if there exist two matrices $M_{2 n \times 2 n}$ and $N_{2 n \times 2 n}$ such that

$$
\begin{gathered}
\text { rank }(M, N)=2 n, \quad M \mathscr{F} M^{T}=N \mathscr{F} N^{T}, \\
T=\left\{\left(y, g^{\pi}\right) \in H(\tau): M\left(\begin{array}{c}
\left(y, \chi_{1}\right)(-\infty) \\
\vdots \\
\left(y, \chi_{2 n}\right)(-\infty)
\end{array}\right)\right. \\
\left.-N\left(\begin{array}{c}
\left(y, \chi_{1}\right)(+\infty) \\
\vdots \\
\left(y, \chi_{2 n}\right)(+\infty)
\end{array}\right)=0\right\} .
\end{gathered}
$$

Remark 43. As we have seen, there is no boundary condition at the endpoints at which system $\left(1_{\lambda}\right)$ is in l.p.c., and the matrix $G_{a}$ or $G_{b}$ can be replaced by $\mathscr{F}$ in the case that system $\left(1_{\lambda}\right)$ is in l.c.c. at $t=a$ or $t=b$.

5.2. At Least One of the Two Endpoints Is Finite. In this subsection, we characterize the $J$-SSEs of $H_{0}(\tau)$ in the special case that at least one of the two endpoints $a$ and $b$ is finite. We first consider the case that $a$ is finite, and $b$ is finite or infinite.

We point out that in this case, characterizations of all the $J$-SSEs of $H_{0}(\tau)$ can also be given by the proof of Theorem 39, provided that assumptions $\left(A_{b, 2}\right),\left(A_{a, 2}\right)$, and $\left(A_{3}\right)$ hold. But, if there does not exist a $c_{0} \in \mathscr{I}$ such that both $\left(A_{a, 2}\right)$ and $\left(A_{b, 2}\right)$ are satisfied, then Theorem 39 fails. We will remark again that the division of $\mathscr{I}$ is not necessary in the case that one of the two endpoints is finite, and characterizations of all the $J$-SSEs of $H_{0}(\tau)$ can still be given provided that $\left(A_{3}\right)$ and $\left(A_{3}\right)$ hold.

In the case that $a$ is finite, $\mathscr{I}$ can be regarded as $\mathscr{I}_{b}$ with $a=c_{0}$, and $\left(A_{2}\right)$ is equivalent to $\left(A_{b, 2}\right)$. So all the characterizations for $H_{b, 0}(\tau)$ and $H_{b}(\tau)$ given in Sections 3 and 4 are available to $H_{0}(\tau)$ and $H(\tau)$, respectively, with $c_{0}$ replaced by $a$. Assume that $\left(A_{2}\right)$ holds. Then for any given $\lambda \in \Gamma\left(H_{0}(\tau)\right)$, as discussed in Section 4 , let $\chi_{1}, \ldots, \chi_{2 d-2 n}$ be $2 d-2 n$ linearly independent solutions in $\mathscr{L}_{W}^{2}(\mathscr{F})$ of $\left(1_{\lambda}\right)$ such that $G$ is invertible, where $G$ is defined by (133) with $\chi_{b}^{j}$ is replaced by $\chi_{j}, 1 \leq j \leq 2 d-2 n$. Then all the results of Theorem 37 hold with $G_{b}$ and $\chi_{b}^{j}$ replaced by $G$ and $\chi_{j}$, respectively.

Theorem 44. Assume that the left endpoint $a$ is finite, and $\left(A_{1}\right)-\left(A_{3}\right)$ hold. Then a subspace $T \subset\left(L_{W}^{2}(\mathscr{I})\right)^{2}$ is a J-SSE of $H_{0}(\tau)$ if and only if there exist two matrices $M_{d \times 2 n}$ and $N_{d \times(2 d-2 n)}$ such that

$$
\begin{aligned}
& \text { (1) } \operatorname{rank}(M, N)=d \text {, } \\
& \text { (2) } M \mathscr{J} M^{T}-N G N^{T}=0 \text {, and }
\end{aligned}
$$

$$
\begin{aligned}
& T=\left\{\left(y, g^{\pi}\right) \in H(\tau): M y(a)\right. \\
&\left.-N\left(\begin{array}{c}
\left(y, \chi_{1}\right)(b+1) \\
\vdots \\
\left(y, \chi_{2 d-2 n}\right)(b+1)
\end{array}\right)=0\right\} .
\end{aligned}
$$

Proof. The main idea of the proof is similar to that of Theorem 39.

We first show the sufficiency. Denote

$$
\mathscr{J} M^{T}=\left(\xi_{1}, \xi_{2}, \ldots, \xi_{d}\right), \quad N=\left(n_{i j}\right)_{d \times(2 d-2 n)^{\prime}}
$$

and set

$$
w_{i}:=\sum_{j=1}^{2 d-2 n} n_{i j} \chi_{j}, \quad 1 \leq i \leq d .
$$


Clearly, $w_{i} \in \operatorname{Dom} H(\tau)$ for $1 \leq i \leq d$. By Remark 26, there exist $\beta_{i}:=\left(\omega_{i}, \rho^{\pi}\right) \in H(\tau)(1 \leq i \leq d)$ such that

$$
\omega_{i}(a)=\xi_{i}, \quad \omega_{i}(t)=w_{i}(t), \quad t \geq t_{0}+1,
$$

where $t_{0}$ is specified by $\left(A_{2}\right)$. It can be verified by the method used in the proof of Theorem 39 that the set $\left\{\beta_{j}\right\}_{j=1}^{d}$ satisfies the conditions (1) and (2) in Lemma 9. Note that for each $y \in$ Dom $H(\tau)$, it follows that

$$
\begin{aligned}
& M y(a)=\left(\mathscr{F} M^{T}\right)^{T} \mathscr{J} y(a) \\
& =\left(\begin{array}{c}
\omega_{1}^{T}(a) \\
\vdots \\
\omega_{d}^{T}(a)
\end{array}\right) \mathscr{J} y(a)=\left(\begin{array}{c}
\left(y, \omega_{1}\right)(a) \\
\vdots \\
\left(y, \omega_{d}\right)(a)
\end{array}\right) \text {, } \\
& N\left(\begin{array}{c}
\left(y, \chi_{1}\right)(b+1) \\
\vdots \\
\left(y, \chi_{2 d-2 n}\right)(b+1)
\end{array}\right) \\
& =\left(\begin{array}{c}
\left(y, \sum_{j=1}^{2 d-2 n} n_{1 j} \chi_{j}\right)(b+1) \\
\vdots \\
\left(y, \sum_{j=1}^{2 d-2 n} n_{d j} \chi_{j}\right)(b+1)
\end{array}\right) \\
& =\left(\begin{array}{c}
\left(y, \omega_{1}\right)(b+1) \\
\vdots \\
\left(y, \omega_{d}\right)(b+1)
\end{array}\right) .
\end{aligned}
$$

Therefore, it follows from Lemma 24 that $T$ can be expressed as

$$
T=\left\{\left(y, g^{\pi}\right) \in H(\tau):\left[\left(y, g^{\pi}\right): \beta_{j}\right]=0,1 \leq j \leq d\right\} .
$$

Hence, $T$ is a $J$-SSE of $H_{0}(\tau)$ by Lemma 9. The sufficiency is proved.

We now show the necessity. Suppose that $T$ is a $J$-SSE of $H_{0}(\tau)$. By Lemma 9 and Theorem 17, there exists a set of $\left\{\beta_{j}\right\}_{j=1}^{d} \subset H(\tau)$ for $\left\{H_{0}(\tau), H(\tau)\right\}$ such that conditions (1) and (2) in Lemma 9 hold, and $T$ can be expressed as (152). Write $\beta_{i}:=\left(\omega_{i}, \rho^{\pi}\right)$. Then $\omega_{j} \in \operatorname{Dom} H(\tau)$ for $1 \leq j \leq d$. By Theorem 37, each $\omega_{i}$ can be uniquely expressed as

$$
\omega_{i}=y_{b}^{i, 0}+\sum_{j=1}^{2 n} a_{i j} z_{b}^{j}+\sum_{s=1}^{2 d-2 n} n_{i s} \chi_{b}^{s}, \quad 1 \leq i \leq d,
$$

where $y_{b}^{i, 0} \in \operatorname{Dom} H_{0}(\tau)$ and $a_{i j}, n_{i s} \in \mathbb{C}$. Set

$$
\begin{aligned}
& M_{d \times 2 n}=\left(\omega_{1}(a), \ldots, \omega_{d}(a)\right)^{T} \mathscr{J}, \\
& N_{d \times(2 d-2 n)}=\left(n_{i s}\right)_{1 \leq i \leq d, 1 \leq s \leq 2 d-2 n} .
\end{aligned}
$$

With a similar argument to that used in the proof of Theorem 39, we can prove that $M$ and $N$ satisfy conditions (1) and (2). In addition, it is clear that $T$ in (170) can be expressed as (165). The necessity is proved, and then the entire proof is complete.
At the end of this subsection, we give the characterizations of $J$-SSEs of $H_{0}(\tau)$ in two special cases of defect indices.

In the special case that $d=n$, Theorem 44 can be described in the following simpler form.

Theorem 45. Assume that the left endpoint $a$ is finite, and $\left(A_{1}\right)-\left(A_{3}\right)$ hold. If $\left(1_{\lambda}\right)$ is in l.p.c. at $t=b$, then a subspace $T \subset\left(L_{W}^{2}(\mathscr{I})\right)^{2}$ is a J-SSE of $H_{0}(\tau)$ if and only if there exists a matrix $M_{n \times 2 n}$ satisfying the self-adjoint condition

$$
\operatorname{rank} M=n, \quad M \mathscr{J} M^{T}=0
$$

such that $T$ can be defined by

$$
T=\left\{\left(y, g^{\pi}\right) \in H(\tau): M y(a)=0\right\} .
$$

In the other special case that $d=2 n$, the following result is a direct consequence of Theorem 44 .

Theorem 46. Assume that the left endpoint $a$ is finite, and $\left(A_{1}\right)-\left(A_{3}\right)$ hold. If $\left(1_{\lambda}\right)$ is in l.c.c. at $t=b$, let $\chi_{i}(1 \leq i \leq$ $2 n)$ be $2 n$ linearly independent solutions in $\mathscr{L}_{W}^{2}(\mathscr{I})$ of $\left(1_{\lambda}\right)$ satisfying $\left(\chi_{1}\left(c_{0}\right), \chi_{2}\left(c_{0}\right), \ldots, \chi_{2 n}\left(c_{0}\right)\right)=I_{2 n}$. Then a subspace $T \subset\left(L_{W}^{2}(\mathscr{I})\right)^{2}$ is a J-SSE of $H_{0}(\tau)$ if and only if there exist two $2 n \times 2 n$ matrices $M$ and $N$ such that

(1) $\operatorname{rank}(M, N)=2 n$,

(2) $M \mathscr{J} M^{T}=N \mathscr{J} N^{T}$, and

$$
\begin{aligned}
& T=\left\{\left(y, g^{\pi}\right) \in H(\tau): M y(a)\right. \\
&\left.-N\left(\begin{array}{c}
\left(y, \chi_{1}\right)(b+1) \\
\vdots \\
\left(y, \chi_{2 n}\right)(b+1)
\end{array}\right)=0\right\} .
\end{aligned}
$$

For the case that $b$ is finite and $a=-\infty$, it can be considered by a similar method. Here we only give the following basic result.

Theorem 47. Assume that the right endpoint $b$ is finite, and $\left(A_{1}\right)-\left(A_{3}\right)$ hold. Let $\chi_{j}, 1 \leq j \leq 2 d-2 n$, be linearly independent solutions of $\left(1_{\lambda}\right)$ in $\mathscr{L}_{W}^{2}(\mathscr{I})$ such that $G$ is invertible, where $G$ is defined as $G_{a}$ in Theorem 38 with $\chi_{a}^{j}$ replaced by $\chi_{j}$. Then a subspace $T \subset\left(L_{W}^{2}(\mathscr{I})\right)^{2}$ is a J-SSE of $H_{0}(\tau)$ if and only if there exist two matrices $M_{n \times 2 n}$ and $N_{d \times(2 d-2 n)}$ such that

(1) $\operatorname{rank}(M, N)=d$,

(2) $M G M^{T}-N \mathscr{J} N^{T}=0$, and

$$
\begin{gathered}
T=\left\{\left(y, g^{\pi}\right) \in H(\tau): M\left(\begin{array}{c}
\left(y, \chi_{1}\right)(a) \\
\vdots \\
\left(y, \chi_{2 d-2 n}(a)\right)
\end{array}\right)\right. \\
-N y(b+1)=0\} .
\end{gathered}
$$


In the case that both the two endpoints $a$ and $b$ are finite, that is, $\mathscr{I}=[a, b]$, it is clear that $d=2 n$ by $\left(A_{2}\right)$. The characterization of $J$-SSEs given in Theorem 44 can be simplified as follows.

Theorem 48. Let $\mathscr{I}=[a, b]$. Assume that $\left(A_{1}\right)-\left(A_{3}\right)$ hold. Then a subspace $T \subset\left(L_{W}^{2}(\mathscr{I})\right)^{2}$ is a J-SSE of $H_{0}(\tau)$ if and only if there exist two $2 n \times 2 n$ matrices $M$ and $N$ such that

(1) $\operatorname{rank}(M, N)=2 n$,

(2) $N \mathscr{J} N^{T}=M \mathscr{J} M^{T}$, and

$$
\begin{gathered}
T=\left\{\left(y, g^{\pi}\right) \in H(\tau): M y(a)\right. \\
-N y(b+1)=0\} .
\end{gathered}
$$

Proof. Let $\chi_{j} \in \mathscr{L}_{W}^{2}(\mathscr{I}), 1 \leq j \leq 2 n$, be defined as those in Theorem 46 . Then it follows that

$$
\begin{aligned}
N\left(\begin{array}{c}
\left(y, \chi_{1}\right)(b+1) \\
\vdots \\
\left(y, \chi_{2 n}\right)(b+1)
\end{array}\right) & =N\left(\begin{array}{c}
\chi_{1}^{T}(b+1) \\
\vdots \\
\chi_{2 n}^{T}(b+1)
\end{array}\right) \mathscr{J} y(b+1) \\
& =N_{1} y(b+1),
\end{aligned}
$$

where $N_{1}=N\left(\chi_{1}, \ldots, \chi_{2 n}\right)^{T}(b+1) \mathscr{J}$. It is evident $\left(\chi_{1}, \ldots\right.$, $\left.\chi_{2 n}\right)(b+1)=I_{2 n}$. So by Lemma 14 , one has that

$$
\begin{gathered}
\operatorname{rank}(M, N)=\operatorname{rank}\left(M, N_{1}\right)=2 n, \\
N_{1} \mathscr{J} N_{1}^{T}=N \mathscr{J} N^{T} .
\end{gathered}
$$

Hence, the assertion follows from Theorem 46. This completes the proof.

\section{Acknowledgment}

This research was supported by the NNSF of China (Grants 11071143, 11101241, and 11226160).

\section{References}

[1] N. I. Akhiezer and I. M. Glazman, Theory of Linear Operators in Hilbert Space, Pitman Publishing, London, UK, 1981.

[2] N. Dunford and J. T. Schwartz, Linear Operators. Part II: Spectral theory. Self Adjoint Operators in Hilbert Space, John Wiley \& Sons, New York, NY, USA, 1963.

[3] I. M. Glazman, Direct Methods of Qualitative Spectral Analysis of Singular Differential Operators, Israel Program for Scientific Translations, Jerusalem, Israel, 1965.

[4] J. Weidmann, Spectral Theory of Ordinary Differential Operators, vol. 1258, Springer, Berlin, Germany, 1987.

[5] H. Sun and Y. Shi, "Self-adjoint extensions for singular linear Hamiltonian systems," Mathematische Nachrichten, vol. 284, no. 5-6, pp. 797-814, 2011.

[6] H. Sun and Y. Shi, "Self-adjoint extensions for linear Hamiltonian systems with two singular endpoints," Journal of Functional Analysis, vol. 259, no. 8, pp. 2003-2027, 2010.
[7] J. Sun, "On the self-adjoint extensions of symmetric ordinary differential operators with middle deficiency indices," Acta Mathematica Sinica, vol. 2, no. 2, pp. 152-167, 1986.

[8] A. Wang, J. Sun, and A. Zettl, "Characterization of domains of self-adjoint ordinary differential operators," Journal of Differential Equations, vol. 246, no. 4, pp. 1600-1622, 2009.

[9] Y. Shi, "The Glazman-Krein-Naimark theory for Hermitian subspaces," Journal of Operator Theory, vol. 68, no. 1, pp. 241256, 2012.

[10] Y. Shi, "Weyl-Titchmarsh theory for a class of discrete linear Hamiltonian systems," Linear Algebra and Its Applications, vol. 416, no. 2-3, pp. 452-519, 2006.

[11] E. A. Coddington, Extension Theory of Formally Normal and Symmetric Subspaces, vol. 134, American Mathematical Society, Providence, RI, USA, 1973.

[12] E. A. Coddington, "Self-adjoint subspace extensions of nondensely defined symmetric operators," Bulletin of the American Mathematical Society, vol. 79, pp. 712-715, 1973.

[13] E. A. Coddington and A. Dijksma, "Self-adjoint subspaces and eigenfunction expansions for ordinary differential subspaces," Journal of Differential Equations, vol. 20, no. 2, pp. 473-526, 1976.

[14] A. Dijksma and H. S. V. de Snoo, "Self-adjoint extensions of symmetric subspaces," Pacific Journal of Mathematics, vol. 54, pp. 71-100, 1974.

[15] M. Lesch and M. Malamud, "On the deficiency indices and self-adjointness of symmetric Hamiltonian systems," Journal of Differential Equations, vol. 189, no. 2, pp. 556-615, 2003.

[16] G. Ren and Y. Shi, "Self-adjoint extensions for discrete linear Hamiltonian systems".

[17] Y. Shi and H. Sun, "Self-adjoint extensions for second-order symmetric linear difference equations," Linear Algebra and its Applications, vol. 434, no. 4, pp. 903-930, 2011.

[18] I. M. Glazman, "An analogue of the extension theory of Hermitian operators and a non-symmetric one-dimensional boundary problem on a half-axis," Doklady Akademii Nauk SSSR, vol. 115 , pp. 214-216, 1957.

[19] D. Race, "The theory of $J$-self-adjoint extensions of $J$-symmetric operators," Journal of Differential Equations, vol. 57, pp. 258-274, 1985.

[20] Z. Shang, "On $J$-self-adjoint extensions ofJ-symmetric ordinary differential operators," Journal of Differential Equations, vol. 73, pp. 153-177, 1988.

[21] S. J. Monaquel and K. M. Schmidt, "On $M$-functions and operator theory for non-self-adjoint discrete Hamiltonian systems," Journal of Computational and Applied Mathematics, vol. 208, no. 1, pp. 82-101, 2007.

[22] H. Sun and J. Qi, “The theory for J-Hermitian subspaces in a product space," ISRN Mathematical Analysis, vol. 2012, Article ID 676835, 16 pages, 2012.

[23] G. Ren and Y. Shi, "Defect indices and definiteness conditions for a class of discrete linear Hamiltonian systems," Applied Mathematics and Computation, vol. 218, no. 7, pp. 3414-3429, 2011. 


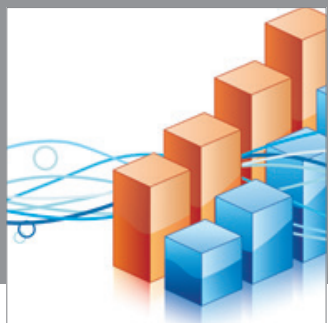

Advances in

Operations Research

mansans

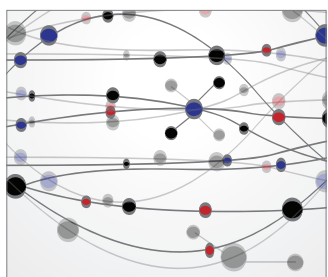

The Scientific World Journal
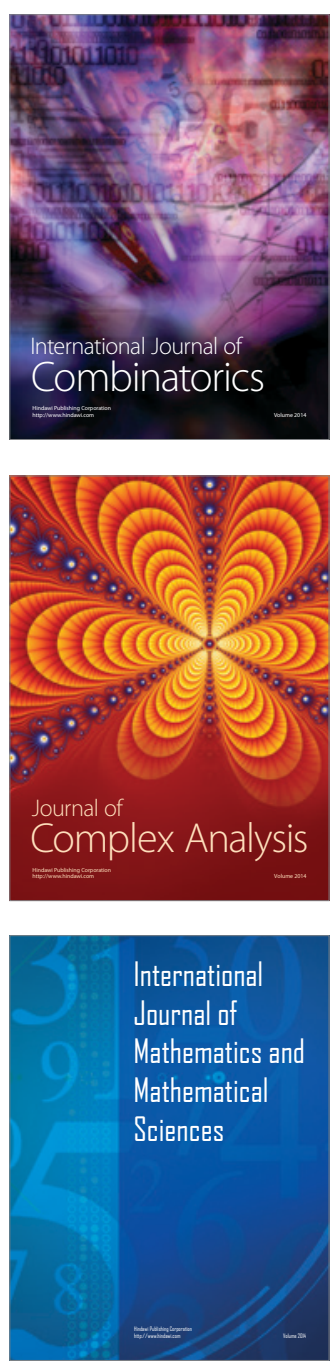
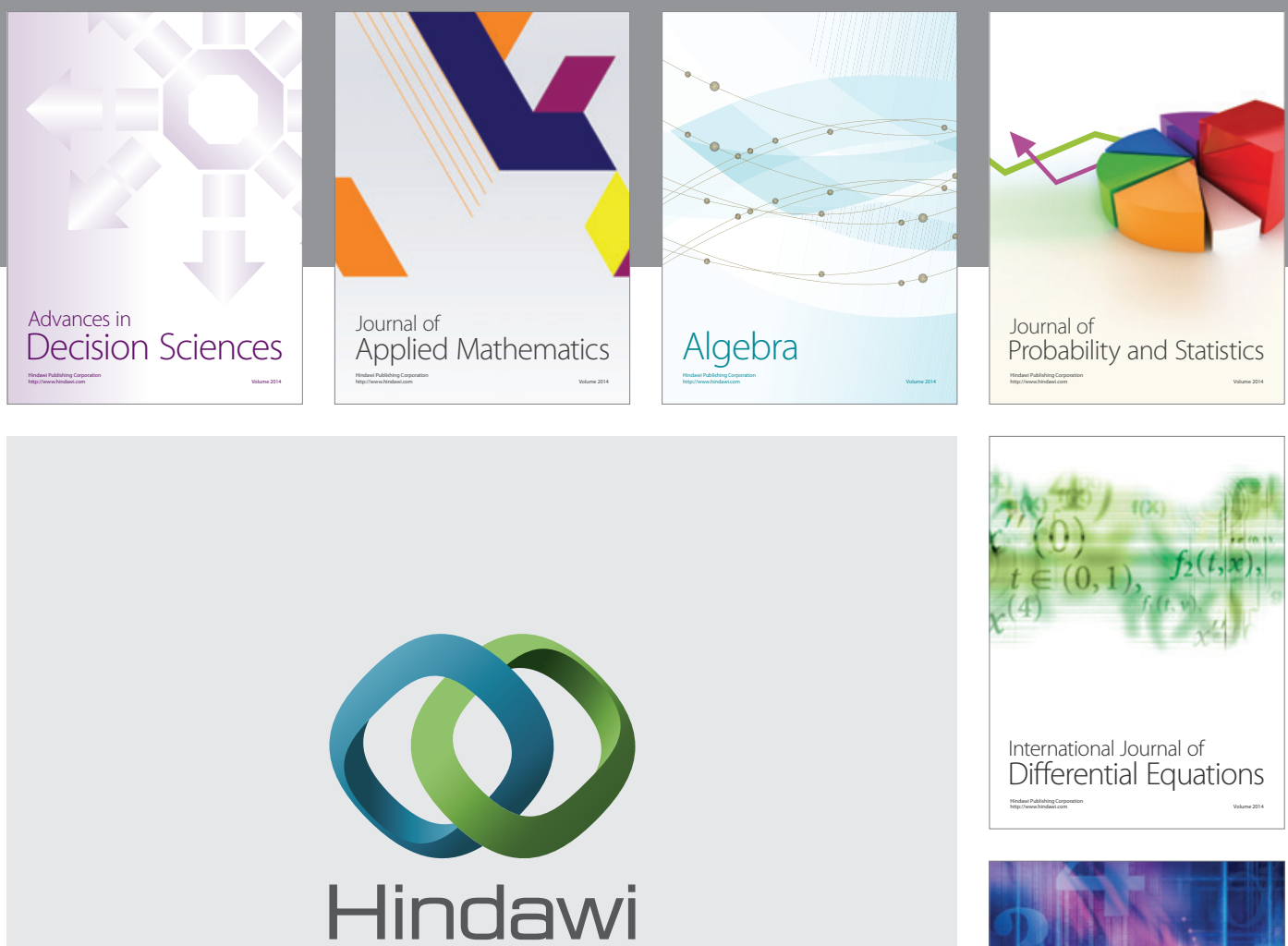

Submit your manuscripts at http://www.hindawi.com
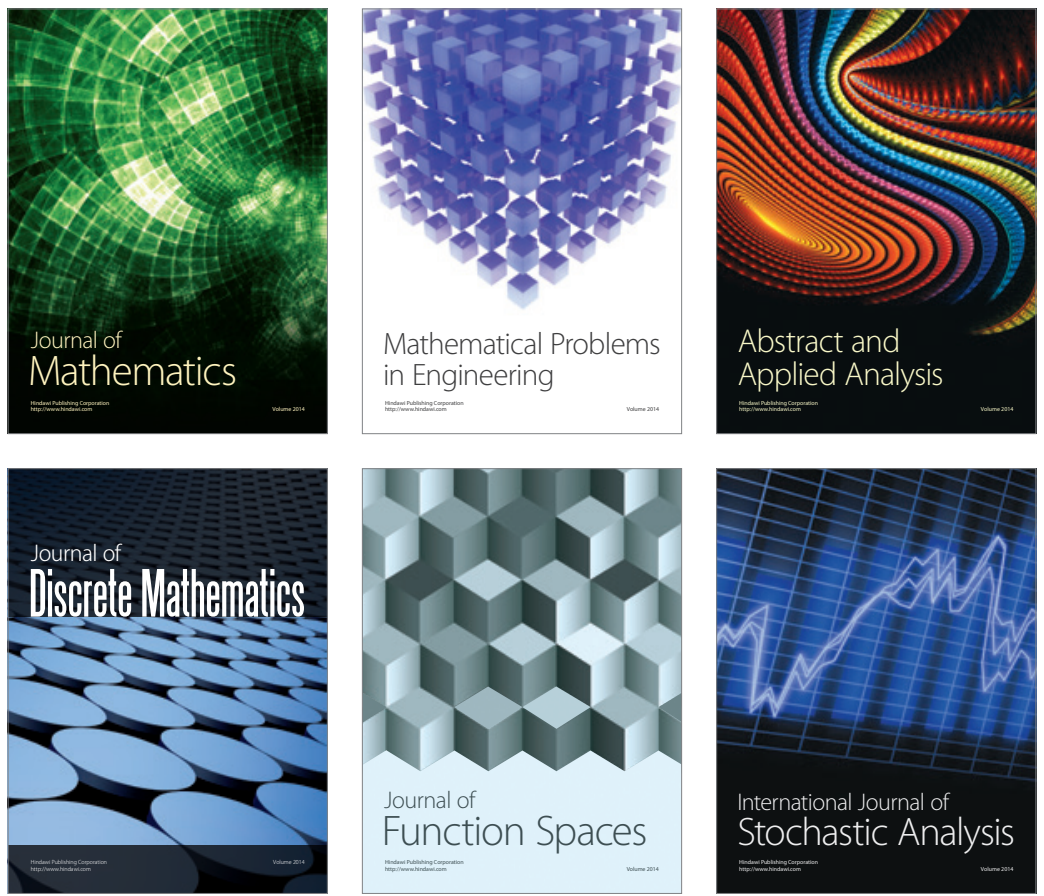

Journal of

Function Spaces

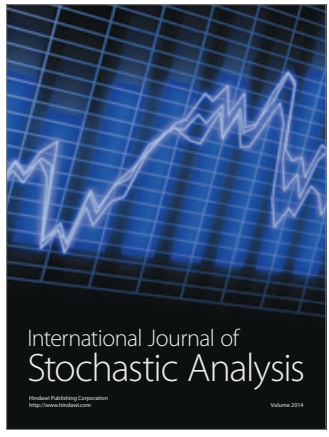

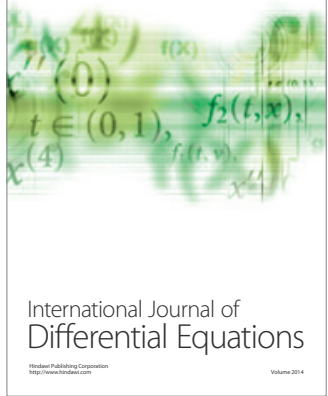
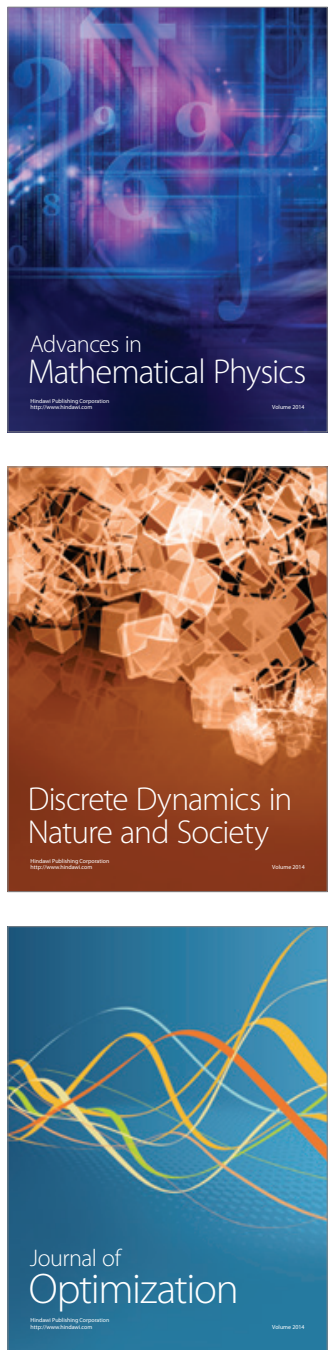